\title{
Improved convergence and stability properties in a three-dimensional higher-order ice sheet model
}

\author{
J. J. Fürst ${ }^{1}$, O. Rybak ${ }^{1}$, H. Goelzer ${ }^{1}$, B. De Smedt ${ }^{1}$, P. de Groen ${ }^{2}$, and P. Huybrechts ${ }^{1}$ \\ ${ }^{1}$ Earth System Sciences \& Department of Geography, Vrije Universiteit Brussel, Pleinlaan 2, Brussels, Belgium \\ ${ }^{2}$ Department of Mathematics, Vrije Universiteit Brussel, Pleinlaan 2, Brussels, Belgium
}

Received: 21 June 2011 - Published in Geosci. Model Dev. Discuss.: 20 July 2011

Revised: 14 October 2011 - Accepted: 12 November 2011 - Published: 19 December 2011

\begin{abstract}
We present a finite difference implementation of a three-dimensional higher-order ice sheet model. In comparison to a conventional centred difference discretisation it enhances both numerical stability and convergence. In order to achieve these benefits the discretisation of the governing force balance equation makes extensive use of information on staggered grid points. Using the same iterative solver, a centred difference discretisation that operates exclusively on the regular grid serves as a reference. The reprise of the ISMIP-HOM experiments indicates that both discretisations are capable of reproducing the higher-order model inter-comparison results. This setup allows a direct comparison of the two numerical implementations also with respect to their convergence behaviour. First and foremost, the new finite difference scheme facilitates convergence by a factor of up to 7 and 2.6 in average. In addition to this decrease in computational costs, the accuracy for the resultant velocity field can be chosen higher in the novel finite difference implementation. Changing the discretisation also prevents build-up of local field irregularites that occasionally cause divergence of the solution for the reference discretisation.

The improved behaviour makes the new discretisation more reliable for extensive application to real ice geometries. Higher accuracy and robust numerics are crucial in time dependent applications since numerical oscillations in the velocity field of subsequent time steps are attenuated and divergence of the solution is prevented.
\end{abstract}

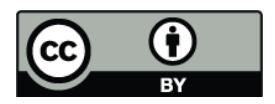

Correspondence to: J. J. Fürst

(johannes.fuerst@vub.ac.be)

\section{Introduction}

Modelling ice sheet evolution is a challenging task since ice flow is controlled by processes that are dominant on various spatial and temporal scales (Vaughan and Arthern, 2007; Truffer and Fahnestock, 2007; Shepherd and Wingham, 2007; Pritchard et al., 2009). Decomposing the inherent complexity of ice dynamics, one can separate two major components: (1) non-slip ice dynamics arising from internal deformation under the ice weight itself and (2) dynamics introduced by lateral and basal boundary conditions. This separation does not mean a superposition of independent components but rather represents a complex system of mutual influence. Especially the second part (2) is controversial since basal processes are not well understood and they are held responsible for major transitions between flow regimes. Key issues at the ice bed are feedbacks between basal hydrology, till deformation and basal ice dynamics (Tulaczyk et al., 2000; Johnson and Fastook, 2002; Pollard and DeConto, 2009; Pimentel et al., 2010). The complexity is further increased by the treatment of the transition between the grounded ice sheet and the floating ice shelves (Schoof, 2007; Goldberg et al., 2009; Gagliardini et al., 2010) and the influence of calving at ice fronts (Benn et al., 2007; Otero et al., 2010; Albrecht et al., 2011). This variety of boundary conditions has a direct impact on pure deformational dynamics, which have to capture strong spatial variations in the ice flow. This is especially the case were regions of ice frozen to bedrock neighbour on areas of sliding (Fowler and Larson, 1980). Since dynamics that arise from ice deformation in turn feed back on basal and lateral conditions, special attention has to be attributed to any simplification applied in the deformational part.

Published by Copernicus Publications on behalf of the European Geosciences Union. 
In general, most large-scale ice sheet models describe ice as a nonlinear viscous and isotropic fluid to capture its deformation. Their main differences lie in the approximations used in the force balance or Stokes equation. The most comprehensive Full-Stokes (FS) models solve the force balance equation without further simplifications to the underlying continuum mechanics (Zwinger et al., 2007; Hindmarsh, 2004; Jouvet et al., 2009). Although they fully capture the deformational dynamics of ice sheets, their applicability is strongly restricted by computational limitations. Therefore approximations derived from scale analysis have been suggeted for ice sheet modelling. On the one hand, the shallow ice approximation (SIA) assumes dominant vertical plane shearing that balances horizontal gradients in the gravity potential (Morland and Johnson, 1980; Hutter, 1983). Apart from clear deficiencies in capturing full ice dynamics, the SIA has proven to be a feasible approach for modelling the evolution of large-scale ice sheets on long time scales (Huybrechts and de Wolde, 1999; Ritz et al., 2001). On the other hand, floating ice shelves show a vertically homogeneous flow with barely any vertical shearing. For ice shelves, dynamics are characterised by so-called membrane stresses (Hindmarsh, 2004, 2006), which are comprised in the shallow shelf approximation (SSA) (Morland, 1986; Weis et al., 1999).

In the vicinity of the transition zones between sliding (also floating) and non-sliding areas, the shallow approximations become inappropriate (Schoof, 2006) and a more comprehensive approach becomes necessary. This gap is filled by models that either superimpose the two shallow approximations (Bueler and Brown, 2009) or by so-called higherorder models (Blatter et al., 1995; Pattyn, 2003; Hindmarsh, 2004; Schoof and Hindmarsh, 2010). In terms of a hierarchy, higher-order models comprise the dynamics of both "shallow" approximations but simplifications to the vertical force balance reduce their complexity compared to FS models. Dukowicz et al. (2010) uses a principle of least action that allows the derivation of all these approximations in one terminology by subsequent simplification. However, the term higher-order model is ambiguous and therefore Hindmarsh (2004) introduced a more rigorous classification. Our higher-order model is classified as including Multilayer Longitudinal Stresses (LMLa), generally referred to as the Blatter/Pattyn approximation (Blatter et al., 1995; Pattyn, 2003). In this approximation the crucial simplification is that in the vertical stress balance so-called bridging terms are neglected meaning a glaciostatic assumption. As a consequence the computation of the vertical velocity field decouples from the dynamic equations and is determined via mass conservation.

The combination of the force balance equation together with a constitutive relation, linking stresses to strain rates, provides a system of partial differential equations that is in general non-linear. Though the LMLa higher-order approximation allows a separate computation of the vertical velocity component, one still has to fully account for the non-linear character of remaining two partial differential equations (PDE) for the horizontal velocity components. Such non-linearity poses a great challenge to any numerical solver. Instead of solving the highly complex system of equations by direct inversion, most solvers work iteratively and start from an initial guess that is updated throughout several iterations until a specified accuracy is reached. Such approaches require a robust numerical implementation of the dynamic equations that prevents oscillation to build up iteratively (Colinge et al., 1998). One way to improve the convergence of an iterative method without changing the numerical discretisation is to introduce an artificial smoothing that attenuates oscillations in intermediate steps. However, the physical interpretation of such smoothed solutions is controversial. Another way is to introduce correction algorithms that optimise the iterative result using information from preceding iterations (Hindmarsh and Payne, 1996; De Smedt et al., 2010). Such algorithms should facilitate convergence while the reduction of iterative steps together with the applied solution correction certainly influence and possibly improve numerical stability.

However, an adjustment of the discretisation of the underlying dynamic equations gives direct control on the consistency of their numerical representation. In case of the higher-order dynamics, the force balance is a partial differential equation of elliptic form. Such PDEs mainly solve stationary problems and are often connected to a minimisation problem (Dukowicz et al., 2010). Especially the numerical theory around the Stokes equation is well established (see Mattheij et al., 2005; Ferziger and Perić, 2002; LeVeque, 2007). Decoupling of the solution in adjacent points using centred differences in the Stokes equation is an understood phenomenon. To increase numerical coupling of the solution in adjacent points, Harlow and Welch (1965) suggest the computation of pressure differences on the regular grid and of velocity gradients on staggered points. A similar approach to couple the solution in adjacent points was suggested by Colinge et al. (1998) who introduced a combination of centred and one-sided derivatives to stabilise the numerics in the original Blatter-type model. Their finite difference scheme substantially reduced the number of iterations necessary to retrieve the solution.

In this work we present and compare a new discretisation variant of the force balance equations of a LMLa higherorder model to the discretisation suggested in Pattyn (2003). In Sect. 2 we clarify our notation and introduce the model equations. We suggest readers familiar with the topic to directly advance to Sect. 2.2 where the novel finite difference scheme is introduced. A short mathematical background is given in 2.3. Section 3 summarises the characteristics of the used numerical solver. Thereafter results from repeating the ISMIP-HOM experiments (Pattyn et al., 2008) are presented for the two discretisations. Finally convergence properties are analysed and compared in Sect. 5. 


\section{Model description}

\subsection{Force balance equation}

For modelling the dynamics of glacial systems we choose an orthogonal coordinate system with three unit vectors $\left\{\boldsymbol{e}_{x}, \boldsymbol{e}_{y}, \boldsymbol{e}_{z}\right\}$ in respectively horizontal $x, y$ and vertical $z$ direction. The vertical axis of our coordinate system is chosen to be perpendicular to isolines of the gravitational field. Our thermo-mechanical approach encompasses two balance equations for mass and momentum combined with a constitutive relation that links the stress tensor $\sigma$ to strain rates $\dot{\boldsymbol{\varepsilon}}$ and thus to the velocity field $\boldsymbol{u}=(u, v, w)$. The ice body is assumed to be incompressible, meaning constant density $\rho$, which causes the balance equations to take the following form.

$$
\begin{aligned}
\partial_{x} u+\partial_{y} v+\partial_{z} w & =0 \\
\rho \cdot \frac{d \boldsymbol{u}}{d t} & =\nabla \sigma-\rho g \cdot \boldsymbol{e}_{z}
\end{aligned}
$$

The gradient operator $\nabla$, being a vector of partial derivatives $\left(\partial_{x}, \partial_{y}, \partial_{z}\right)$, is applied to the stress tensor. The mean density of the ice body is represented by $\rho$ while the gravitational acceleration is given by $g$. The acceleration term in the force balance equation is in general omitted but not, as sometimes stated, because it is negligibly small. On the contrary, accelerations in fact reach large values but the time needed to adjust the velocity field and attain a new balance of forces is small. Moreover, accelerations do not trigger dominant dynamic feedback that decisively influences the overall force balance. On relevant time scales in glaciology accelerations consequently become negligible. The stresses of the resultant force balance are in turn related to the strain rate $\dot{\boldsymbol{\varepsilon}}$ via a constitutive relation for the creep of polycrystalline ice. Here Nye's generalisation of Glen's flow equation is chosen:

$\tau_{i j}=2 \eta \dot{\varepsilon}_{i j}$

$\eta=\frac{1}{2} A_{0}^{-1 / n}\left(\dot{\varepsilon}_{e}+\dot{\varepsilon}_{0}\right)^{1 / n-1}$

where $\boldsymbol{\tau}_{i j}$ are deviatoric stresses, $n$ is the Glen index and $A_{0}$ is a rate factor. The positive scalar $\eta$ is the effective viscosity, which is defined via the second invariant of the strain rate $\dot{\boldsymbol{\varepsilon}}_{e}^{2}=\frac{1}{2} \sum_{x, y, z} \dot{\varepsilon}_{i j} \dot{\varepsilon}_{i j}$ making it independent of the particular coordinate system. The strain rate tensor is the link between the applied forces and the response of the material in terms of velocity gradients $\dot{\varepsilon}_{i j}=\frac{1}{2}\left(\partial_{i} u_{j}+\partial_{j} u_{i}\right)$. Following Pattyn (2003) a negligible offset $\varepsilon_{0}^{2}=10^{-30} \mathrm{yr}^{-2}$ is used to prevent singularities in the viscosity field $\eta$ (for $n>1$ ) beneath the ice divide.

The comprehensive set of Eqs. (1)-(4) is simplified to obtain a LMLa higher-order model (cf. Hindmarsh, 2004) following the notation in Pattyn (2003). The glaciostatic approximation, we may neglect any bridging effects (i.e. horizontal gradients of the vertically directed shearing field $\left.\partial_{x} \boldsymbol{\tau}_{x z}, \partial_{y} \boldsymbol{\tau}_{y z} \ll \partial_{z} \boldsymbol{\tau}_{z z}\right)$ in the force balance (2). This is combined with the assumption that horizontal gradients in the vertical velocity field are negligible in comparison with vertical gradients of horizontal velocities $\left(\partial_{x} w \ll \partial_{z} u, \partial_{y} w \ll\right.$ $\left.\partial_{z} v\right)$. Together these two approximations cause a decoupling of the horizontal velocity field from the vertical one that can diagnostically be determined via mass conservation (1). They reduce the applicability of the LMLa model to ice geometries with large horizontal extent compared to their thickness. Additionally, the vertical coordinate is normalised by the ice thickness $H$ using the following coordinate transformation: $(x, y, \zeta) \rightarrow\left(x^{\prime}, y^{\prime}, \zeta\right)$ with $\zeta=(s-z) / H$ and $s$ is the surface elevation. The force balance in $x$-direction is then rewritten in a form analogous to Eq. (44) in Pattyn (2003)

$$
\begin{gathered}
4 \cdot \partial_{x^{\prime}}\left(\eta \partial_{x^{\prime}} u\right)+4 a_{x} \cdot \partial_{x^{\prime}}\left(\eta \partial_{\zeta} u\right)+4 a_{x} \cdot \partial_{\zeta}\left(\eta \partial_{x^{\prime}} u\right)+ \\
\partial_{y^{\prime}}\left(\eta \partial_{y^{\prime}} u\right)+a_{y} \cdot \partial_{y^{\prime}}\left(\eta \partial_{\zeta} u\right)+a_{y} \cdot \partial_{\zeta}\left(\eta \partial_{y^{\prime}} u\right)+ \\
\left(4 a_{x}^{2}+a_{y}^{2}+a_{z}^{2}\right) \cdot \partial_{\zeta}\left(\eta \partial_{\zeta} u\right)+\left(4 b_{x}+b_{y}\right) \eta \cdot \partial_{\zeta} u \\
= \\
\rho g \cdot \partial_{x^{\prime}} s-2 \cdot \partial_{x^{\prime}}\left(\eta \partial_{y^{\prime}} v\right)-\partial_{y^{\prime}}\left(\eta \partial_{x^{\prime}} v\right)- \\
2 a_{y} \cdot \partial_{x^{\prime}}\left(\eta \partial_{\zeta} v\right)-2 a_{x} \cdot \partial_{\zeta}\left(\eta \partial_{y^{\prime}} v\right)-3 a_{x} a_{y} \cdot \partial_{\zeta}\left(\eta \partial_{\zeta} v\right)- \\
a_{x} \cdot \partial_{y^{\prime}}\left(\eta \partial_{\zeta} v\right)-a_{y} \cdot \partial_{\zeta}\left(\eta \partial_{x^{\prime}} v\right)-3 c_{x y} \eta \cdot \partial_{\zeta} v .
\end{gathered}
$$

The coefficients $a_{x}, a_{y}, b_{x}, b_{y}$ and $c_{x y}$ are defined via the coordinate transformation and details are given in Appendix A. In the $y$-direction the force balance takes an analogue form. The effective viscosity in turn takes the following form.

$$
\begin{aligned}
\eta= & \frac{1}{2} A_{0}^{-1 / n}\left\{\left(\partial_{x^{\prime}} u+a_{x} \cdot \partial_{\zeta} u\right)^{2}+\left(\partial_{y^{\prime}} v+a_{y} \cdot \partial_{\zeta} v\right)^{2}+\right. \\
& \left(\partial_{x^{\prime}} u+a_{x} \cdot \partial_{\zeta} u\right) \cdot\left(\partial_{y^{\prime}} v+a_{y} \cdot \partial_{\zeta} v\right)+ \\
& \frac{1}{4}\left(\partial_{y^{\prime}} u+a_{y} \cdot \partial_{\zeta} u+\partial_{x^{\prime}} v+a_{x} \cdot \partial_{\zeta} v\right)^{2}+ \\
& \left.\frac{a_{z}^{2}}{4}\left(\partial_{\zeta} u\right)^{2}+\frac{a_{z}^{2}}{4}\left(\partial_{\zeta} v\right)^{2}+\dot{\varepsilon}_{0}\right\}^{1 / n-1}
\end{aligned}
$$

In order to find a unique solution for Eqs. (5) and (6), boundary conditions are required. At the ice-free points around the lateral boundary we set not only the ice thickness $H$ to zero but also the velocity field. This Dirichlet boundary condition is widely used in ice sheet modelling although the resulting margin gradients are thus dictated by grid spacing. The upper surface is assumed to be stress free. This implies (Van der Veen, 1999)

$0=\left.\left[\left(4 \cdot \partial_{x} u+2 \cdot \partial_{y} v\right) \cdot \partial_{x} s+\left(\partial_{y} u+\partial_{x} v\right) \cdot \partial_{y} s-\partial_{z} u\right]\right|_{s}$,

evaluated at the surface $(z=s)$. The ice-bed contact however provides resistance and a similar boundary condition arises with an additional friction term.

$\tau_{\mathrm{b} x}=\left.\left[\tau_{x z}-\tau_{x y} \cdot \partial_{y} \mathrm{~b}-\left(2 \cdot \tau_{x x}+\tau_{y y}\right) \cdot \partial_{x} \mathrm{~b}\right]\right|_{\mathrm{b}}$.

The basal resistance $\tau_{\mathrm{b} x}$ is assumed to follow a linear law for frictional sliding (MacAyeal, 1989). The basal velocity is hereby linked via a "frictional coefficient" $\beta^{2}$ to basal resistance $\tau_{\mathrm{b} x}, \tau_{\mathrm{b} y}$.

$$
\tau_{\mathrm{b} x}=-\left.\beta^{2} \cdot u\right|_{\mathrm{b}}, \quad \tau_{\mathrm{b} y}=-\left.\beta^{2} \cdot v\right|_{\mathrm{b}}
$$


Since the new discretisation is applied for the bulk Eq. (5), no-slip conditions $\left(u_{\mathrm{b}}=v_{\mathrm{b}}=0\right)$ are assumed for the moment. To retrieve the vertical velocity, one makes use of the incompressibility Eq. (1) and integrates it from the ice sheet base $\mathrm{b}$ to $z$.

In the following, Eq. (5) is rewritten such that most terms are replaceed by two operators. Except for three terms, all summands contain a derivative of the product of an effective viscosity with an inner velocity derivative. Making use of this similarity, we introduce two operators $\Omega, \Psi$ that act on $k$ times continuously differentiable functions $f, g \in C^{k}\left(\mathbb{R}^{3}\right)$.

$$
\begin{aligned}
& \Omega(s, g, f) \equiv \partial_{s}\left(g \cdot \partial_{s} f\right) \\
& \Psi(s, t, g, f) \equiv \partial_{s}\left(g \cdot \partial_{t} f\right)
\end{aligned}, s \neq t \text { with } s, t \in\left\{x^{\prime}, y^{\prime}, \zeta\right\}
$$

With them Eq. (5) can be rewritten as follows.

$$
\begin{aligned}
& 4 \cdot \Omega\left(x^{\prime}, \eta, u\right)+4 a_{x} \cdot \Psi\left(x^{\prime}, \zeta, \eta, u\right)+4 a_{x} \cdot \Psi\left(\zeta, x^{\prime}, \eta, u\right)+ \\
& \Omega\left(y^{\prime}, \eta, u\right)+a_{y} \cdot \Psi\left(y^{\prime}, \zeta, \eta, u\right)+a_{y} \cdot \Psi\left(\zeta, y^{\prime}, \eta, u\right)+ \\
& \left(4 a_{x}^{2}+a_{y}^{2}+a_{z}^{2}\right) \cdot \Omega(\zeta, \eta, u)+\left(4 b_{x}+b_{y}\right) \eta \cdot \partial_{\zeta} u \\
& = \\
& \rho g \cdot \partial_{x^{\prime}} s-2 \cdot \Psi\left(x^{\prime}, y^{\prime}, \eta, v\right)-\Psi\left(y^{\prime}, x^{\prime}, \eta, v\right)- \\
& 2 a_{y} \cdot \Psi\left(y^{\prime}, \zeta, \eta, v\right)-2 a_{x} \cdot \Psi\left(\zeta, y^{\prime}, \eta, v\right)- \\
& 3 a_{x} a_{y} \cdot \Omega(\zeta, \eta, v)-a_{x} \cdot \Psi\left(y^{\prime}, \zeta, \eta, v\right)- \\
& a_{y} \cdot \Psi\left(\zeta, x^{\prime}, \eta, v\right)-3 c_{x y} \eta \cdot \partial_{\zeta} v .
\end{aligned}
$$

The uniformity introduced by these two operators can be exploited both on the level of discretisation and of programming. By analogy the force balance in $y$-direction is rewritten in terms of these two operators.

\subsection{Numerical realisation of main operators}

In order to solve Eqs. (5), (6) and (7) on a fixed grid, we consider two finite difference schemes. On the one hand a "direct" discretisation (DIR) is used that approximates any derivative with a centred difference scheme. This DIR scheme exclusively uses information on the regular grid as suggested in Pattyn (2003). The non-uniform vertical grid spacing is treated by centred differences that are weighted with coefficients derived from Newton's polynomial approximation formula (see Appendix B, Eqs. B8 and B9). On the other hand, a second discretisation is presented that uses information at staggered grid points (STAG) to compute the two operators.

Equations (6) and (7) show exclusively first derivatives. Those are approximated with second-order centred differences in both, DIR and STAG. The surface boundary condition (7) is treated in exactly the same way with the vertical gradient being calculated by a one-sided difference scheme of second order. Although centred differences are applied in both discretisations, effective viscosities (6) are computed at different points. While DIR defines $\eta$ on regular points, STAG computes them in the grid box centre (see Fig. 1). Consequently the centred differences in DIR cover twice the grid spacing while STAG operates only on one grid cell. Since velocities are defined on the regular grid, the STAG

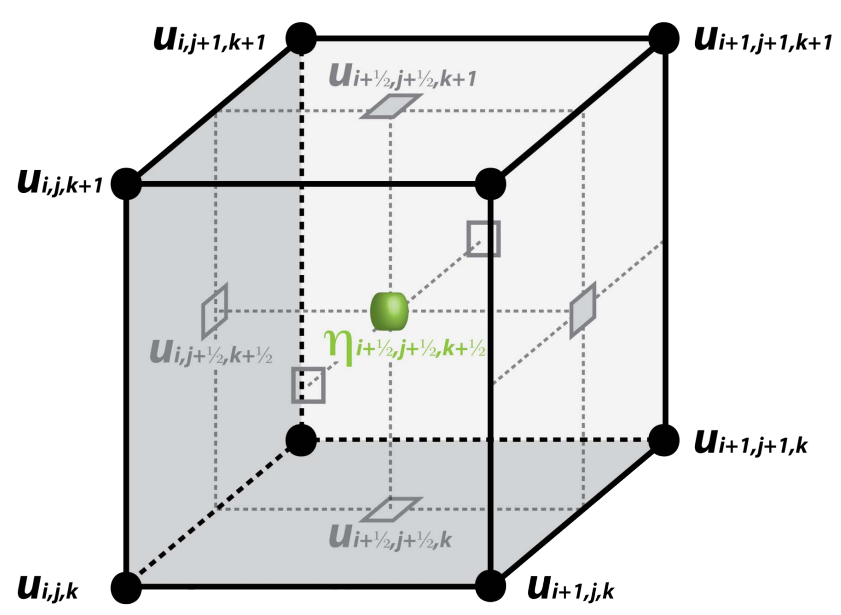

Fig. 1. Grid cell as used in the STAG implementation. Note that velocities are defined at grid points while effective viscosities are computed in the centre. To determine necessary velocity derivatives one first computes the velocity on each face of the grid box.

scheme needs the average of four at each side of the box. Subsequently derivatives are calculated using values at opposite faces of the cell, i.e. the derivative in $x$-direction requires the velocity field averaged in the $y, \zeta$-plane. The STAG discretisation of the effective viscosity thereby reduces the truncation error of the velocity derivatives with respect to DIR. Note however that the truncation error is not necessarily a good indicator for the accuracy of a discrete solution (Veldmann and Rinzema, 1992) and in particular not for a nonuniform grid spacing.

The main difference between the two schemes lies in the discretisation of the force balance Eqs. $(5,11)$. This applies to the finite difference approximation used for the two operators $\Omega ; \Psi$. The three additional terms, that show mere first derivatives either in velocities or surface elevation, do not show the structure necessary for $\Omega, \Psi$. Consequently a distinct discretisation is used, which we base on centred differences between the two inline-adjacent grid points (analogous to DIR). The two discretisations thus ultimately differ in the realisation of the two operators $\Omega, \Psi$ that are defined via

$$
\begin{gathered}
\Omega(s, g, f) \equiv \partial_{s}\left(g \cdot \partial_{s} f\right)=\partial_{s} g \cdot \partial_{s} f+g \cdot \partial_{s}^{2} f \\
\Psi(s, t, g, f) \equiv \partial_{s}\left(g \cdot \partial_{t} f\right)=\partial_{s} g \cdot \partial_{t} f+g \cdot \partial_{s} \partial_{t} f
\end{gathered}
$$

In the DIR scheme, operators are rewritten using the chain rule (right hand side of Eq. 10) and the resultant summands show derivatives that are approximated by centred differences of first order. This DIR discretisation is presented in detail in Pattyn (2003). It accepts that the discretisation of $\eta$ leaves the compact stencil. In contrast to applying the chain rule in DIR, the STAG scheme profits from the compact definition of $\Omega, \Psi$. The terms in brackets, being input for the outer derivative, are computed at staggered points. For this the effective viscosity and the inner derivative need to 
be determined on the relevant, staggered position for a centred difference approximation of the outer derivative. With the finite differences spanning only one grid cell, the STAG scheme shows naturally a reduced truncation error.

A detailed description of the STAG scheme, giving the full decomposition in finite differences, is provided in Appendix B. In this section a more heuristic description is presented to clarify the fundamental steps. To illustrate them, we focus on the horizontal operator $\Psi(x, y, \eta, u)=\partial_{x}\left(\eta \cdot \partial_{y} u\right)$, which has the advantage to act on an equidistant mesh. The term in brackets, still undergoing the derivative in $x$, is determined in between adjacent grid points in $x$-direction. This allows a central difference approximation of the $x$-derivative on half the grid spacing. For this purpose both the velocity derivative in $y$-direction and the effective viscosity need to be determined at the relevant position. As illustrated in Fig. 2, this is achieved in four steps.

1. First, one linearly averages adjacent velocities to get values in between grid points in $x$-direction $u_{i+\frac{1}{2}, j}$.

2. With $u_{i+\frac{1}{2}, j}$, the centred derivative in $y$-direction is calculated giving $\left(\partial_{y} u\right)_{i+\frac{1}{2}, j+\frac{1}{2}}$ in the middle of $x, y$ faces. This derivative is then multiplied with a viscosity average $\left(\eta \cdot \partial_{y} u\right)_{i+\frac{1}{2},{ }_{j}+\frac{1}{2}}$ of two adjacent grid cell centres.

3. Subsequent computation of the centred derivative in $x$-direction of $\left(\eta \cdot \partial_{y} u\right)_{i+\frac{1}{2}, j+\frac{1}{2}}$ provides $\partial_{x}(\eta$. $\left.\partial_{y} u\right)_{i, j+\frac{1}{2}} \cdot$

4. Averaging the derivative field $\partial_{x}\left(\eta \cdot \partial_{y} u\right)_{i, j+\frac{1}{2}}$ linearly in $y$-direction, one obtains it on the regular grid (cf. Fig. 2).

Swapping $x$ and $y$, the operator $\Psi(y, x, \eta, u)$ is determined in an analogue way. Derivatives operating on the vertical axis hold an additional complexity since equal spacing in this direction is not granted. For non-uniform spacing, weighting coefficients are used that are based on Newton's formula for a second-order polynomial approximation (see Eqs. B8 and B9). These two equations can be applied in analogy to the above discussion to determine the STAG version of $\Psi(\zeta, x, \eta, u), \Psi(\zeta, y, \eta, u), \Psi(x, \zeta, \eta, u)$ and $\Psi(y, \zeta, \eta, u)$.

For $\Omega$ operators that show two derivatives in the same direction, one determines the inner derivative in between adjacent grid points with centred differences. This derivative is then multiplied by a four-point average of viscosities that gives $(\eta \cdot \partial u)$ in between adjacent, in-line grid points. The outer derivative is then determined centred over half the grid spacing. A detailed description of the numerical implementation is presented in Appendix B.

In summary, we have two discretisations of the force balance equation. First the DIR scheme that is based on centred

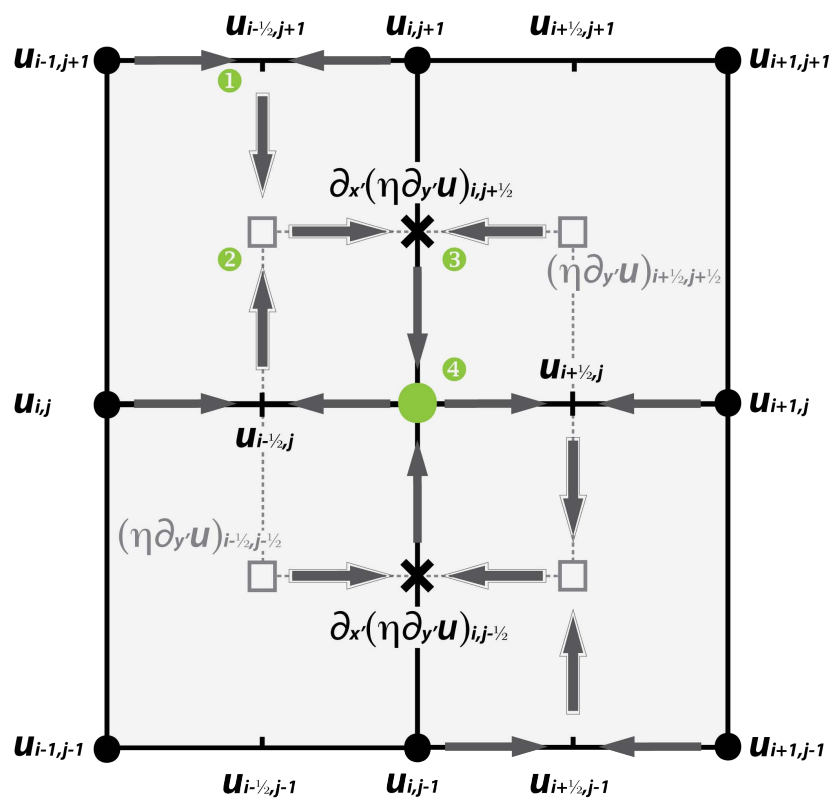

Fig. 2. Visualization of the operating mode of $\Psi(x, y, u, \eta)$. In a first step, two adjacent velocities are averaged in $x$-direction. This field is then used to form the inner derivative in $y$-direction that is associated with the grid point staggered in the $x, y$-plane (step 2). After determining the viscosity in this point, the outer $x$-derivative can be computed in a third step. The fourth and last step is to average the resultant field in $x$-direction (a detailed description is presented in Appendix B).

differences for the fully decomposed operators (cf. Pattyn, 2003). As shown, this scheme is not confined to the compact stencil for the bulk equation. The STAG discretisation is confined to the compact stencil and makes use of information on staggered positions. In this way it increases coupling of the velocity solution in adjacent grid points and reduces the truncation error.

\subsection{Operator invertibility}

In mathematical terms, a structural difference is present in the two ways of discretising the $\Omega$ operator. Consider the following ordinary differential equation in one dimension

$\Omega(x, \eta, u)=\partial_{x}\left(\eta \cdot \partial_{x} u\right)=f$,

with $f \in C\left(\mathbb{R}^{3}\right)$. To numerically approximate the solution we choose a one dimensional grid with uniform spacing $\Delta x$. The DIR discretisation makes use of the chain rule and approximates the two resulting terms with centred differences.

$\left(-\eta_{i}-\partial_{x} \eta_{i} \Delta x\right) \cdot u_{i+1}+\left(-\eta_{i}+\partial_{x} \eta_{i} \Delta x\right) \cdot u_{i-1}+2 \eta_{i} \cdot u_{i}=-f_{i} \Delta x^{2}$

In the STAG scheme, $\left(\eta \cdot \partial_{x} u\right)_{i+1 / 2}$ is determined at staggered points. This information can in turn enter directly a 
centred difference approximation for the outer derivative on half the grid extent.

$$
-\eta_{i+\frac{1}{2}} \cdot u_{i+1}-\eta_{i-\frac{1}{2}} \cdot u_{i-1}+\left(\eta_{i+\frac{1}{2}}+\eta_{i-\frac{1}{2}}\right) \cdot u_{i}=-f_{i} \Delta x^{2}
$$

Despite the inherent dependence of the viscosity $\eta$ on the velocity field, we will treat it for now as a given scalar field. Since the viscosity is by definition positive (see Eq. 6), the factors of the diagonal element are positive in both numerical schemes. Both discretisations also result in a matrix $\mathbf{M}$ of tridiagonal form. However, the STAG discretisation provides additional structure to $\mathbf{M}$ because it guarantees that the off diagonal entries are all negative. In the DIR scheme this is only true as long as $\eta_{i} \geq\left\|\Delta x \cdot \partial_{x} \eta_{i}\right\|$. The resultant matrix structure for STAG ensures the invertibility of $\mathbf{M}$ since an additional requirement holds which states the existence of a vector $\boldsymbol{u}$ with positive components $u_{i}>0$ that fulfils $(\mathbf{M} \boldsymbol{u})_{i}>0$ (in literature such matrices are referred to as $\mathbf{M}$ matrices and the interested reader is referred to Berman and Plemmons, 1994). For the DIR scheme, invertibility is not guaranteed especially for large grid spacing or high gradients in the viscosity field. The STAG discretisation will consequently stabilise any numerical method that determines the inverse of such a linear system of equations. In other words, the STAG discretisation provides a matrix $\mathbf{M}$ that unconditionally inherits a discrete maximum principle from the continuous operator, whereas DIR does so only for sufficiently small mesh sizes.

In addition, if one resolves the velocity dependence of the viscosity for DIR, one notices that the $\Omega$ operator leaves the compact stencil during each non-linear iteration step. This is caused by the appearance of the first viscosity derivative which itself uses centred differences for the velocity gradients. It can give rise to spurious oscillations in the solution, especially in the vicinity of the lateral boundaries. This is also the case for the discretisation of the $\Psi$ operator. In contrast to DIR, the STAG discretisation for both operators is always confined to the compact stencil of adjacent grid points.

\section{Iterative solver}

\subsection{Decomposition of the non-linear system of equations}

We reduce the complexity of the nonlinear system by decomposing Eq. (11) into coupled linear equations following Pattyn (2003). The key is to iteratively update the effective viscosity that is nonlinearly dependent on the horizontal velocity field (see Eq. 6). For each nonlinear iteration step the viscosity field is prescribed. Doing so, the determination of the horizontal velocity field from the force balance Eq. (11) becomes a linear problem. In other words, one prescribes an initial velocity field, computes the resulting viscosity field and subsequently determines the velocity solution to the force balance. Subsequently one enters the next nonlinear step and updates the viscosity field that is in turn fed into the linear system.

\subsection{Linear iteration}

For solving the linear system of equations represented by Eq. (11), another decomposition is made separating the two horizontal components of the velocity vector $u$ and $v$. More precisely, knowing both velocity components from the previous nonlinear iteration, one uses them to retrieve the respective perpendicular component in the current iteration $r$. Thus, the current ${ }^{r} \tilde{u}$ becomes a function of both the previous viscosity ${ }^{r-1} \eta$ and the previous $y$-component of the velocity field ${ }^{r-1} v$. This leads to a numerical decoupling of the $x$ and $y$-direction of the force balance equation in the nonlinear iterations and consequently reduces the matrix size of the linear system by a factor 4 . Using one of the two discretisations of the force balance Eq. (11), one can rewrite them in matrix form assuming the respective perpendicular velocity component as given.

$$
\begin{aligned}
& \Lambda_{x}\left({ }^{r-1} \eta\right){ }^{r} \tilde{u}=\boldsymbol{B}_{x}\left({ }^{r-1} u,{ }^{r-1} v\right) \\
& \Lambda_{y}\left({ }^{r-1} \eta\right)^{r} \tilde{v}=\boldsymbol{B}_{y}\left({ }^{r-1} u,{ }^{r-1} v\right)
\end{aligned}
$$

The so-called coefficient matrices $\Lambda$ are sparse in both discretisations since only a few off-diagonal elements are nonzero. Using a grid of respectively $n_{x}, n_{y}, n_{\zeta}$ points in the $x-, y-$ and $\zeta$-direction, the matrix has $N \times N$ entries with $N=n_{x} \cdot n_{y} \cdot n_{\zeta}$. Among all matrix coefficients, at most 19 out of $N$ are non-zero in each matrix column. This ratio falls below one percent for grids consisting of more than 2000 points. Linear systems of equations that show highly sparse matrices are efficiently solved iteratively. One prominent solver for such systems is the bi-conjugate gradient (BiCG) method (Press et al., 2003) with a diagonal preconditioner. Iterative methods use in general criteria that define saturated convergence. A build-in criterion is applied that successively computes the ratio of two maximum norms. The numerator shows the precondition matrix applied on the residual while the denominator holds the current correction vector (see Press et al., 2003). A relative tolerance $\varepsilon_{\text {lin }}$ defines the stopping criterion for which convergence is reached.

\subsection{Non-linear iteration}

As already discussed above, the non-linear iterations update the viscosity field and thus simplify the force balance to a linear system. However, since the viscosity holds the full nonlinearity, its values cover several orders of magnitude over an entire ice sheet. This is a challenge for the convergence of the linear solver and a potential cause for instability. For the results presented here, we use a direct Picard iteration to substitute successive solutions. This means that one substitutes the solution from the linear solver directly ${ }^{r} u={ }^{r} \tilde{u}$. However there are methods to improve the convergence rate (e.g. Hindmarsh et al., 1996; De Smedt et al., 2010) using 
Table 1. Overview of model parameters used in the ISMIP-HOM benchmark (Pattyn et al., 2008).

\begin{tabular}{llrl}
\hline Symbol & Description & Value & Unit \\
\hline$A_{0}$ & $\begin{array}{l}\text { Rate factor in the flow } \\
\text { equation }\end{array}$ & $1.14 \cdot 10^{-5}$ & $\mathrm{~Pa}^{-3} \mathrm{a}^{-1}$ \\
$g$ & $\begin{array}{l}\text { Gravitational acceleration } \\
\text { constant }\end{array}$ & 9.81 & $\mathrm{~m} \mathrm{~s}^{-2}$ \\
$\rho$ & $\begin{array}{l}\text { Average density of ice body } \\
\text { Exponent in Glen's flow } \\
\text { equation }\end{array}$ & 910 & $\mathrm{~kg} \mathrm{~m}^{-3}$ \\
& & 3 & - \\
\hline
\end{tabular}

information from the previous solution ${ }^{r} u=f\left({ }^{r} \tilde{u},{ }^{r-1} u\right)$. Although such correction schemes may be beneficial, they perturb a clear comparison of the two numerical schemes and are therefore omitted. Instead, true Picard iterations are used until the ratio of the Euclidean norm of the residual and the norm of the solution between successive iterations falls below a certain threshold $\varepsilon_{\text {nin }}$. The two criteria $\varepsilon_{\text {lin }}$ and $\varepsilon_{\text {nin }}$ define the quality of the solution and are referred to as the convergence tolerance or simply the tolerance.

\section{Model intercomparison and validation}

In the following, results are presented from conducting experiments of the ISMIP-HOM model intercomparison study (Pattyn et al., 2008). First the experimental setup is specified that serves to evaluate the two discretisations.

In the framework of this intercomparison study four out of six time independent experiments were selected. The remaining two add no additional challenge since they are flowband versions of two others. Test A is a purely geometric problem with an inclined surface topography, frozen bed and sinusoidal bed topography. In test $\mathrm{C}$, a tilted slab of ice with constant thickness is forced with a sinusoidal sliding field $\beta^{2}$ at the base (see Eq. 9). Experiments $\mathrm{A}$ and $\mathrm{C}$ use periodic boundary conditions at the lateral domain margin. In addition, both experiments are conducted for several aspect ratios, i.e. the ratio of ice thickness and domain extent. This ratio is linked to the length scale of the sinusoidal perturbations. Test E2 and E1 are applications on the observed geometry of Haut Glacier d'Arolla respectively with and without a zone of zero friction. The details to each experimental setup are found in Pattyn et al. (2008) while the suggested model parameters are summarised in Table 1. All experiments were conducted on a grid with 100 equally spaced points in both horizontal directions and 100 exponentially spaced layers in the vertical. For Haut Glacier d'Arolla (exp. E1, E2) the horizontal dimension across the flow line was reduced to 83 . In all experiments the shallow ice approximation serves to calculate an initial velocity field. The criteria to stop our iterative solver, i.e. the convergence tolerance, are set equal for both linear and non-linear iterations $\varepsilon_{\text {nin }}=\varepsilon_{\text {lin }}=\varepsilon$.
Although the two discretisations yield different solutions to these experiments (Fig. 3), they both reproduce the results of the intercomparison study. Differences become more evident when the aspect ratio decreases (Fig. $3 b$ and c). However, results are in general within the root mean square (rms) deviation of the higher-order model participants of ISMIPHOM to their average. In the combined geometric and basal boundary problem of test E2, the difference between the STAG and DIR schemes is largest while DIR exceeds the rms deviation, which is large by itself. The fact that solutions for E2 show such a high variation is due to the fact that the setup with a zone of no friction might actually be ill posed (see Pattyn et al., 2008). In light of this a direct comparison of the solution is possibly inappropriate. In contrast to some model participants of the E2 experiment, both discretisation schemes suggest smooth solutions suppressing build-up of oscillations where the base supports no resistance. Experiments $\mathrm{A}$ and $\mathrm{C}$ were also conducted on the $20 \mathrm{~km}$ domain to check the applicability on intermediate aspect ratios. The resultant velocity fields (not shown) are also in qualitative agreement with the ISMIP-HOM results. Except for experiment E2, no major qualitative differences in the solutions are perceived. In Sect. 5.3 non-physical irregularities in the solution are discussed which are observed in experiment A on a $160 \mathrm{~km}$ domain.

\section{Numerical characteristics}

The focus of this section is to compare the two discretisations by concentrating on the characteristics of convergence. First of all, the residual between successive velocity fields during the non-linear iterations is used to validate the quality of the convergence. In a second step the total amount of linear iterations needed to converge is analysed in both numerical schemes and in a final step, we assess the numerical stability of the discretisation.

\subsection{Residual decrease}

The norm of the residual between successive solutions for the velocity field during iteration is referred to as the iteration accuracy. This is a scale free scalar that has to fall below the prescribed tolerance value $\varepsilon_{\text {nin }}$ to reach convergence. With progressive iteration number, the magnitude of the accuracy decreases for most experiments regularly in both discretisations (Fig. 4b). But with smaller tolerance convergence becomes more erratic for the DIR scheme and the residual norm shows large values even in the last few non-linear steps (Fig. 4a). This spurious behaviour indicates not attenuated oscillations in the velocity fields of consecutive iterations. As a consequence the attainable accuracy in the DIR scheme is reduced. Curiously these oscillations are even observed in experiment A that uses prescribed zero velocities at the base. Such spurious behaviour is not observed in any converged experiment for STAG, where convergence is more regular. 

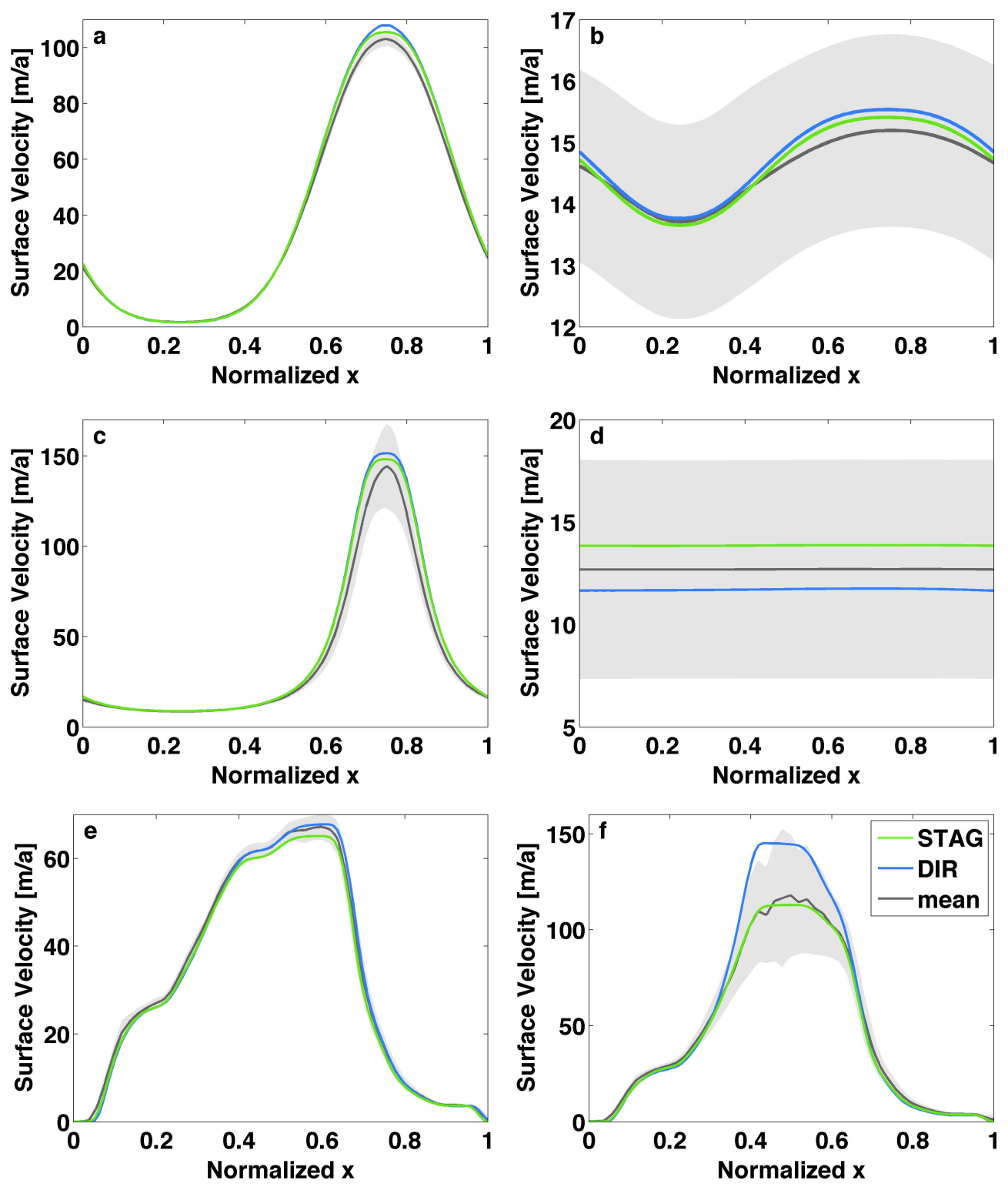

Fig. 3. Resultant surface velocity fields for the ISMIP-HOM model intercomparison of the DIR and STAG discretisations for experiments A on $160 \mathrm{~km} \mathrm{(a)} \mathrm{and} 5 \mathrm{~km}(\mathbf{b}), \mathrm{C}$ on $160 \mathrm{~km}$ (c) and $5 \mathrm{~km}$ (d) and E1 (e) and E2 (f). The grey shaded area indicates the root mean square (rms) deviation of the solution from each participant compared to the mean benchmark solution (dark grey). For the two discretisations, we chose the solution obtained with the highest possible convergence accuracy (see Table 2). All over the results are in good agreement with the mean of the benchmark test. The only exception is experiment E2 (f) where the DIR velocity field exceeds the wide rms deviation.

\subsection{Convergence rate}

A mere comparison of the iteration accuracy, being determined in the non-linear iteration, does not capture the convergence behaviour in the inner, linear iterations. It is the number of linear iterations that holds information about the actually undertaken calculations and thus the computational costs for the convergence (see Table 2). The convergence tolerance comprises the two thresholds $\varepsilon=\varepsilon_{\text {lin }}=\varepsilon_{\text {nin }}$ and it is varied in a range from $10^{-3}$ to $10^{-6}$. Tolerance is defined to be smaller for lower values of $\varepsilon$. The main conclusion drawn from Table 2 is that STAG is computationally more efficient than DIR. In other words, the ratio between the number of linear iterations of STAG and DIR for each experimental setup is larger than 1 . Thus the STAG discretisation allows the solver to retrieve the solution for a specific tolerance in less iterations. Furthermore, the STAG scheme can often determine the solution more precisely than DIR. This is less pronounced in purely geometric problems. But as soon as basal sliding is allowed, convergence in the DIR scheme is 
Table 2. Convergence behaviour of the DIR and the STAG discretisation for the ISMIP-HOM experiments. The convergence tolerance is equal for linear and non-linear iterations and varied from $10^{-3}$ to $10^{-6}$. Note that the number of linear iterations is proportional to the computational costs. "•" signifies that the accuracy could not be reached and the convergence was manually stopped after an reasonable number of non-linear iterations. Divergence of the resultant velocity field is marked with "o". If a certain tolerance was not reached in either way, the same experiment was not conducted with higher accuracy (“-”).

\begin{tabular}{|c|c|c|c|c|}
\hline & Conv. Tolerance $\varepsilon$ & $\begin{array}{r}\text { DIR } \\
\text { Total Linear Iterations }\end{array}$ & $\begin{array}{r}\text { STAG } \\
\text { Total Linear Iterations }\end{array}$ & Ratio \\
\hline TEST A & $10^{-3}$ & 416 & 208 & 2.00 \\
\hline \multirow[t]{3}{*}{$160 \mathrm{~km}$} & $10^{-4}$ & 4614 & 1709 & 2.70 \\
\hline & $10^{-5}$ & 23702 & 6170 & 3.84 \\
\hline & $10^{-6}$ & $\bullet$ & 14441 & - \\
\hline TEST A & $10^{-3}$ & 4561 & 3368 & 1.35 \\
\hline \multirow[t]{3}{*}{$20 \mathrm{~km}$} & $10^{-4}$ & 21822 & 19012 & 1.15 \\
\hline & $10^{-5}$ & 513685 & 115197 & 4.46 \\
\hline & $10^{-6}$ & $\bullet$ & 404369 & - \\
\hline TEST A & $10^{-3}$ & 14578 & 9410 & 1.55 \\
\hline \multirow[t]{3}{*}{$5 \mathrm{~km}$} & $10^{-4}$ & 97372 & 81879 & 1.19 \\
\hline & $10^{-5}$ & 595019 & 280740 & 2.12 \\
\hline & $10^{-6}$ & $\bullet$ & 975601 & - \\
\hline TEST C & $10^{-3}$ & 4047 & 2312 & 1.75 \\
\hline \multirow[t]{3}{*}{$160 \mathrm{~km}$} & $10^{-4}$ & ○ & 16126 & - \\
\hline & $10^{-5}$ & - & 34377 & - \\
\hline & $10^{-6}$ & - & 75399 & - \\
\hline TEST C & $10^{-3}$ & 11853 & 10870 & 1.32 \\
\hline \multirow[t]{2}{*}{$20 \mathrm{~km}$} & $10^{-4}$ & 71088 & 52227 & 1.36 \\
\hline & $10^{-5}$ & $\bullet$ & $\bullet$ & - \\
\hline TEST C & $10^{-3}$ & 18033 & 4376 & 4.12 \\
\hline \multirow[t]{3}{*}{$5 \mathrm{~km}$} & $10^{-4}$ & ○ & 44008 & - \\
\hline & $10^{-5}$ & - & 1862887 & - \\
\hline & $10^{-6}$ & - & $\bullet$ & - \\
\hline \multirow[t]{4}{*}{ TEST E1 } & $10^{-3}$ & 4160 & 1178 & 3.53 \\
\hline & $10^{-4}$ & 5494 & 4221 & 1.30 \\
\hline & $10^{-5}$ & $\circ$ & 12572 & 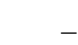 \\
\hline & $10^{-6}$ & - & 33901 & - \\
\hline \multirow[t]{4}{*}{ TEST E2 } & $10^{-3}$ & 7204 & 916 & 7.86 \\
\hline & $10^{-4}$ & ○ & 4580 & - \\
\hline & $10^{-5}$ & - & 30710 & - \\
\hline & $10^{-6}$ & - & 82160 & - \\
\hline
\end{tabular}

only reached for tolerances up to $10^{-4}$. Knowing that a tolerance of at least $10^{-4}$ is necessary to guarantee saturated convergence, this is a grave restriction for the application of the DIR scheme. However, even in cases where the prescribed tolerance could not be reached, we stopped the solver manually after a specific amount of non-linear iterations. This arbitrary number was estimated consulting the non-linear steps for the same setup with less stringent tolerance together with assessing the actual accuracy decrease during convergence. In these not-converged experiments, the effectively reached accuracy is not necessarily higher than for the last converged state of the same experiment setup since $\varepsilon$ influences the two convergence criteria in the linear and non-linear part of the solver. However, most of the not-converged velocity 

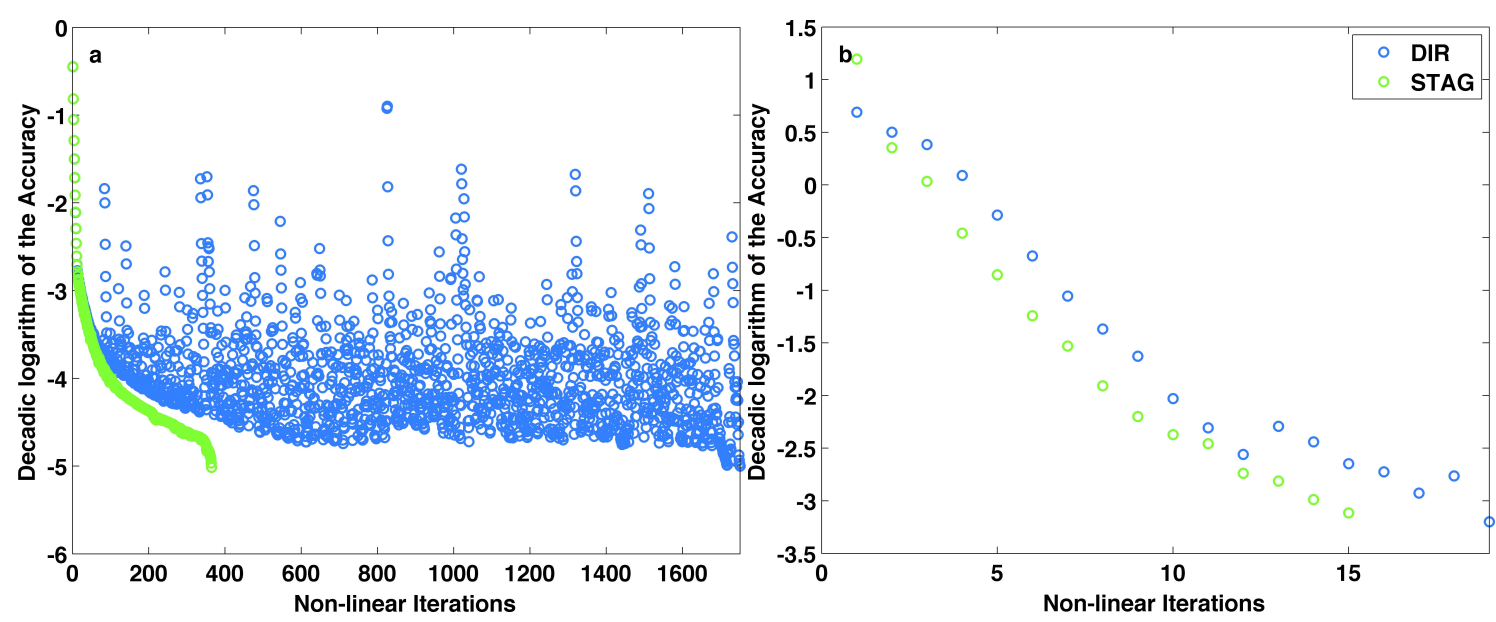

Fig. 4. The residual between successive velocity solutions (i.e. the accuracy) is depicted throughout the non-linear iterations for both discretisations. On the left (a) the convergence of the ISMIP-HOM test A on a $20 \mathrm{~km}$ domain is shown. The decrease in DIR is erratic and indicates huge changes in the velocity field even in the last few iteration steps. For the same setup, the STAG scheme is characterised by a more regular decrease in the accuracy until the prescribed tolerance of $10^{-5}$ is reached. In addition, less non-linear iterations are necessary to retrieve the solution as is confirmed in test $\mathrm{C}$ on $20 \mathrm{~km}$ (b), where both discretisations show a more regular convergence.

solutions remain in agreement with the ISMIP-HOM results. Anyway a lack in convergence raises concerns about the applicability of the retrieved solution.

\subsection{Numerical stability}

In all of the conducted ISMIP-HOM intercomparison experiments (Pattyn et al., 2008) the STAG scheme gives physically reasonable results, though in two cases not strictly converged. But STAG shows robust convergence that prevents possible divergence of the resultant velocity field (see Table 2 and Fig. 4). Divergence only occurs for the DIR scheme and the retrieved flow field deviates by orders of magnitude from the physically reasonable one. The diverged solution exhibits jagged structures that indicate numerical instability, which occurs preferentially for sliding experiments that apply Neuman boundary conditions at the base. But also for a setup without sliding, DIR diverges as soon as a realistic and rugged geometry is used (compare experiment E1). Divergent behaviour is critical since it makes transient experiments almost impossible because the velocity solution might destabilise for any realised geometry during the time evolution. To circumvent this problem in DIR, a smoothing algorithm was applied on the viscosity field (results not shown). In detail, $\eta$ was linearly interpolated on the centre of the grid box and subsequently interpolated back on the regular grid. This prevented divergence of the resultant velocity field in the DIR scheme. But this smoothing did neither facilitate nor inhibit the convergence process. Moreover, no consistent improvement in the attainable accuracy for the DIR discretisation could be stated. For these reasons, such a smoothing algorithm seems capable to prevent divergence but it appears to neither facilitate the convergence rate nor allow for higher accuracies of the solution. Moreover, solutions from a solver using artificial smoothing are delicate to interpret since they might miss crucial details of the dynamic behaviour.

A more detailed examination of the resultant surface velocity fields of the ISMIP-HOM test A reveals for coarse resolutions a qualitative difference between the two discretisations. On the $160 \mathrm{~km}$ domain, the maximal velocity decreases and seems to saturate with increasing accuracy (Fig. 5). Remarkable is however a feature appearing in the DIR discretisation. For high tolerances, the maximum becomes locally flat and even shows a local depression for $\varepsilon=10^{-5}$ (see Fig. 5a). This is in contradiction with properties of the solution to the elliptic force balance equations (cf. Eq. 5). For this low aspect ratio, velocities are dominated by vertical plane shearing and magnitudes are comparable with the solution of the shallow ice approximation. In such a situation Appendix $\mathrm{C}$ suggets that local extrema in the horizontal velocity field are ultimately linked to extrema in the basal topography. A local minimum in bedrock elevation thus goes along with one local maximum in the velocity field. Identifying three adjacent local extrema in the DIR velocity field indicates that its discretisation breaks properties of the solution to an elliptic PDE as the force balance Eq. (5). At least, these properties are not captured in this specific example with coarse resolution. Existence of spurious extrema can also be a seed for destabilisation during the iterative process of determining the solution.

\section{Summary and outlook}

In this study we compare two numerical discretisations of the force balance Eq. (5) used in a Blatter/Pattyn a higher-order 

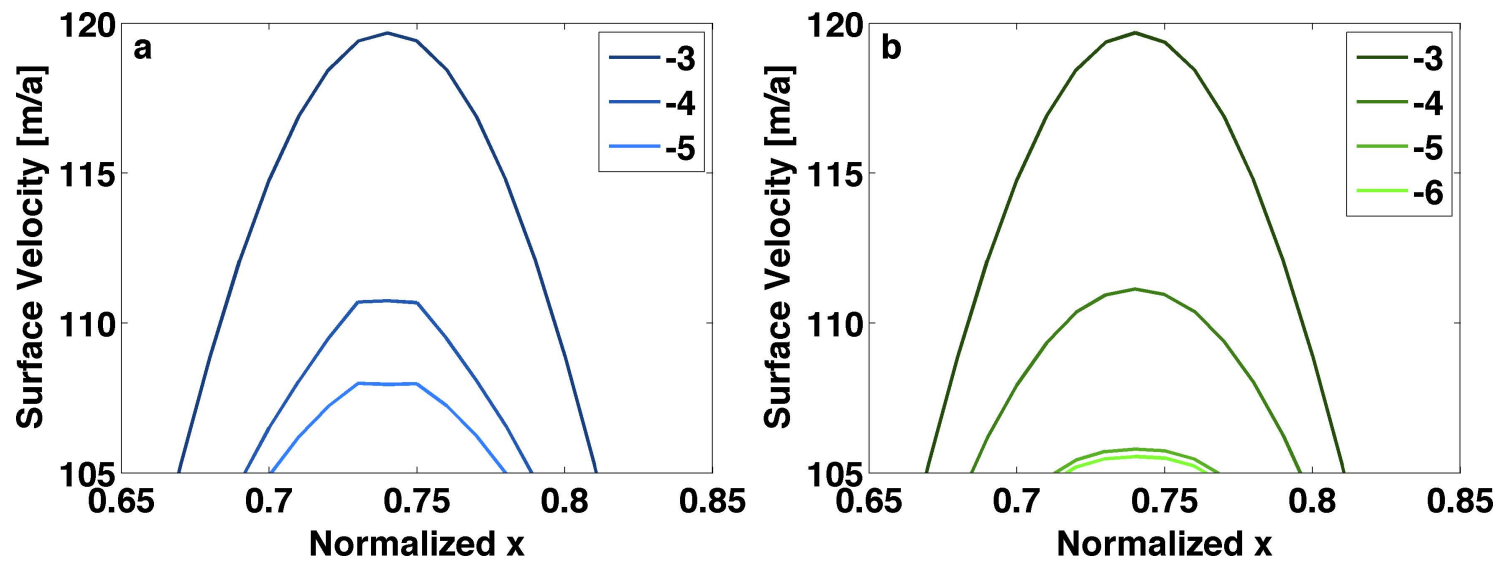

Fig. 5. Resultant surface velocity fields in the ISMIP-HOM test A on the largest domain of $160 \mathrm{~km}$ for the DIR discretisation (a) and the STAG discretisation (b). This is a close-up view of the zone of high deformation where the $x$-component of the bedrock gradient changes its sign. The legend entries refer to different convergence tolerances expressed in the exponent of 10 . For a low tolerance, both discretisations find a solution close to the initial SIA velocity (not depicted). With increasing accuracy the resultant velocity fields converge to a solution with lower maxima. However, the DIR field levels out at the maxima and even shows a local minimum for the highest accuracy.

ice sheet model. We use a LMLa higher-order model in the notation of Hindmarsh (2004) that applies some simplification to the full Stokes equation. The first discretisation, referred to as the DIR scheme, uses the chain rule to decompose all terms in the force balance to substitute each by centred difference, as suggested by Pattyn (2003). The presented new STAG discretisation makes use of the present double derivatives and computes the terms necessary for the outer derivative on staggered grid points (see Fig. 2).

In general both discretisations reproduce the results presented in the ISMIP-HOM model intercomparison study (Pattyn et al., 2008). The good agreement between the two discretisations in the resultant velocity fields encourages a clean comparison of their convergence behaviour. Altogether, we note that the new scheme facilitates the convergence and reduces the total amount of iterations by a factor of up to 7 and in average 2.6 (see Table 2). This implies a decrease in iterative calculations making STAG computationally more efficient. Another benefit using the new discretisation becomes apparent for increased convergence tolerance. In most conducted experiments the STAG solution can attain a higher accuracy than possible in the DIR discretisation. This indicates that erratic build-up of oscillations between successive velocity fields is prevented. Spurious oscillations entering the periodic boundary conditions will even more deteriorate the solution of the linear part of the solver and inhibit convergence for high tolerance. The attainable higher tolerance for the STAG solution therefore indicates more robust numerics. For experiment $\mathrm{A}$ on the coarsest resolution, the DIR surface velocity field along a flow line shows three adjacent local extrema in the zone of fastest flow. For this experiment, such features are per se not feasible in the solution to the elliptic, partial differential equation for the force balance. Additionally, such local irregularities can be a seed for destabilisation of the iterative process. In four out of 18 experiments destabilisation even causes divergence of the DIR velocity field throughout the iterations. Consequently no physical velocity solution is found for a specific experimental setup. Such deficiency is not observed in the STAG scheme indicating robustness of its numerical discretisation, which prevents the iterative build up of perturbations.

Increased convergence rate, higher accuracy and prevention of divergence in the STAG discretisation make the presented discretisation more reliable for any application on observed or artificial geometries. Especially in time-dependent mode, with prognostic evolution of the ice geometry, divergence in the velocity solution would pose a huge problem. Being more robust the new discretisation stabilises the transient behaviour. But not only a converged velocity field is important in time dependency. Also accuracy is decisive since low accuracy results in not fully converged velocity fields and consequently an inadequate geometry evolution. These inaccuracies in turn feed back on the velocity field of the next time step and thus transmit in a prognostic way. Only high accuracy guarantees physically correct feedbacks between higher order dynamics and geometry evolution in transient applications.

Although the new discretisation facilitates convergence, the increase in calculation speed is only a welcome side effect. To decrease computational costs significantly we suggest the application of other mathematical solvers as since $\mathrm{BiCG}$ is known to exhibit erratic convergence behaviour. A stabilised version of Bi-CG (BiCGstab) was suggested by Van der Vorst (1992) and its convergence rate was improved with $\mathrm{ML}(\mathrm{K})-\mathrm{BiCG}$ stab applying a Lanczos process using multiple starting left Lanczos vectors (Yeung et al., 1999). But there are also other techniques to solve nonsymmetric systems of linear equations as the generalized 
minimal residual method (GMRES in Saad and Schultz, 1986), multigrid approaches (see Wesseling and Sonneveld, 1980; Trottenberg et al., 2001), the induced dimension reduction (IDR) method (Sonneveld and van Gijzen, 2008; van Gijzen and Sonneveld, 2011) or a combination of IDR and BiCGstab called IDRstab (Sleijpen and van Gijzen, 2010). In line with replacing the solver is the use of a more appropriate preconditioner for matrix inversion for incompressible fluid dynamics (see Manguoglu et al., 2009). Another option is to combine the applied solver with some correction algorithm that improves the convergence rate. A useful relaxed Picard iteration was presented in De Smedt et al. (2010). An additional improvement to the presented discretisation could be expected by a strict separation of geometry on regular points and velocity on staggered points as suggested by Harlow and Welch (1965). More recently Hyman et al. (2002) suggest a mimetic finite difference method for diffusion problems (as the $\Omega$ operator) that satisfies conservation laws and is applicable for nonsmooth and unstructured grids. In case Neumann boundary conditions are used at the base, a revision of the centred discretisation of the boundary layer equation may give additional numerical benefits.

\section{Appendix A}

A dimensionless vertical coordinate system is used that normalises the vertical axis via $\zeta=(s-z) / H$. This results in a vertical coordinate that varies from zero at the surface $s$ to one at the glacier bed $b$. Since the two horizontal axes remain unchanged, the Jacobian of this coordinate transformation to $\left(x^{\prime}, y^{\prime}, \zeta\right)$ takes a simple form (also compare Pattyn, 2003). For a function $f=f(x, y, z)$ in the class of $k$ times continuous differentiable functions $C^{k}\left(\mathbb{R}^{3}\right)$ (for $k \geq 2$ ) this yields

$$
\begin{aligned}
& \partial_{x} f=\partial_{x^{\prime}} f+a_{x} \cdot \partial_{\zeta} f \\
& \partial_{y} f=\partial_{y^{\prime}} f+a_{y} \cdot \partial_{\zeta} f \\
& \partial_{z} f=a_{z} \cdot \partial_{\zeta} f
\end{aligned}
$$

while the coefficients denote the non-zero elements of the Jacobian.

$$
\begin{aligned}
& a_{x}=\partial_{x^{\prime}} \zeta=\frac{1}{H}\left(\partial_{x^{\prime}} s-\zeta \cdot \partial_{x^{\prime}} H\right) \\
& a_{y}=\partial_{y^{\prime}} \zeta=\frac{1}{H}\left(\partial_{y^{\prime}} s-\zeta \cdot \partial_{y^{\prime}} H\right) \\
& a_{z}=\partial_{z} \zeta=-\frac{1}{H}
\end{aligned}
$$

However, the balance Eq. (5) also shows second order derivatives in all variables. Only four of the possible second derivates actually occur.

$$
\begin{aligned}
\partial_{x x} f= & \partial_{x^{\prime} x^{\prime}} f+b_{x} \cdot \partial_{\zeta} f+a_{x}^{2} \cdot \partial_{\zeta \zeta} f+2 a_{x} \cdot \partial_{x^{\prime} \zeta} f \\
\partial_{y y} f= & \partial_{y^{\prime} y^{\prime}} f+b_{y} \cdot \partial_{\zeta} f+a_{y}^{2} \cdot \partial_{\zeta \zeta} f+2 a_{y} \cdot \partial_{y^{\prime} \zeta} f \\
\partial_{z z} f= & a_{z}^{2} \cdot \partial_{\zeta \zeta} f \\
\partial_{x y} f= & \partial_{x^{\prime} y^{\prime}} f+c_{x y} \cdot \partial_{\zeta} f+a_{y} \cdot \partial_{x^{\prime} \zeta} f+a_{y} \cdot \partial_{y^{\prime} \zeta} f+ \\
& a_{x} a_{y} \cdot \partial_{\zeta \zeta} f,
\end{aligned}
$$

For these derivatives the coefficients are defined as follows

$$
\begin{aligned}
& b_{x}=\partial_{x^{\prime}} a_{x}+a_{x} \cdot \partial_{\zeta} a_{x} \\
& b_{y}=\partial_{y^{\prime}} a_{y}+a_{y} \cdot \partial_{\zeta} a_{y} \\
& c_{x y}=\partial_{x^{\prime}} a_{y}+a_{x} \cdot \partial_{\zeta} a_{y} .
\end{aligned}
$$

\section{Appendix B Discretisation of bulk equation}

This section deals with the detailed description of the numerical discretisation of the bulk Eq. (11). In the following a short overview is given how the two operators $\Omega, \Psi$ are numerically realised for an equidistant mesh as well as for a non-equidistant mesh with weighting based on Newton's parabolic interpolation formula.

\section{B1 Equidistant mesh}

In the horizontal plane the adjacent grid points have uniform spacing $(\Delta x, \Delta y)$. Thus horizontal derivatives can directly be translated via centred differences. It is sufficient to present the operators $\Omega(x, \eta, u)$ and $\Psi(y, x, \eta, u)$ since the other two horizontal operators follow in analogy.

\section{B1.1 In-line derivative $\Omega(x, \eta, u)$}

The in-line derivative $\Omega(x, \eta, u)$ is formed by determining the inner term $\eta \cdot \partial_{x} u$ in the centre between to adjacent points in $x$. These are subsequently used to determine the outer centred derivative. In detail this reads.

$$
\begin{aligned}
& \eta_{i+1 / 2, j}\left(\frac{\partial u}{\partial x}\right)_{i+\frac{1}{2}, j}=\frac{1}{2 \Delta x}\left[\left(u_{i+1, j}-u_{i, j}\right) \cdot\left(\eta_{i+\frac{1}{2}, j+\frac{1}{2}}+\eta_{i+\frac{1}{2}, j-\frac{1}{2}}\right)\right] \\
& \eta_{i-1 / 2, j}\left(\frac{\partial u}{\partial x}\right)_{i-\frac{1}{2}, j}=\frac{1}{2 \Delta x}\left[\left(u_{i, j}-u_{i-1, j}\right) \cdot\left(\eta_{i-\frac{1}{2}, j+\frac{1}{2}}+\eta_{i-\frac{1}{2}, j-\frac{1}{2}}\right)\right]
\end{aligned}
$$

In the vertical both terms are determined on grid layers (index omitted) and, since viscosities are defined in grid box centres, one has to average on layer $\xi_{k}$.

$$
\begin{array}{r}
\eta_{i+\frac{1}{2}, j+\frac{1}{2}}=\frac{\zeta_{k}-\zeta_{k-1}}{\zeta_{k+1}-\zeta_{k-1}} \cdot \eta_{i+\frac{1}{2}, j+\frac{1}{2}, k+\frac{1}{2}}+ \\
\frac{\zeta_{k+1}-\zeta_{k}}{\zeta_{k+1}-\zeta_{k-1}} \cdot \eta_{i+\frac{1}{2}, j+\frac{1}{2}, k-\frac{1}{2}}
\end{array}
$$

Knowing the inner derivative in between adjacent points in $x$-direction, the outer $x$-derivative of $\Omega(x, \eta, \boldsymbol{u})$ is conveniently approximated by a centred difference.

$$
\begin{aligned}
\frac{\partial}{\partial x}\left[\eta\left(\frac{\partial u}{\partial x}\right)\right]_{i, j}=\frac{1}{\Delta x}[ & \left.\eta_{i+\frac{1}{2}, j}\left(\frac{\partial u}{\partial x}\right)_{i+\frac{1}{2}, j}-\eta_{i-1 \frac{1}{2}, j}\left(\frac{\partial u}{\partial x}\right)_{i-\frac{1}{2}, j}\right]= \\
=\frac{1}{2 \Delta x^{2}}[ & u_{i+1, j}\left(\eta_{i+\frac{1}{2}, j+\frac{1}{2}}+\eta_{i+\frac{1}{2}, j-\frac{1}{2}}\right)+ \\
& u_{i-1, j}\left(\eta_{i-\frac{1}{2}, j+\frac{1}{2}}+\eta_{i-\frac{1}{2}, j-\frac{1}{2}}\right)- \\
& \left.u_{i, j}\left(\eta_{i+\frac{1}{2}, j+\frac{1}{2}}+\eta_{i+\frac{1}{2}, j-\frac{1}{2}}+\eta_{i-\frac{1}{2}, j+\frac{1}{2}}+\eta_{i-\frac{1}{2}, j-\frac{1}{2}}\right)\right]
\end{aligned}
$$

\section{B1.2 Cross derivative $\Psi(y, x, \eta, u)$}

Numerically these two perpendicular derivatives are determined within the compact stencil in the $x, y$-plane. The first step (cf. Fig. 1) is to average the regular grid velocities in 
$y$-direction. A centred derivative in $x$ of this averaged velocity field defines $\left(\partial_{x} u\right)_{i+\frac{1}{2}, j+\frac{1}{2}}$, staggered in the $x-$ and $y$-direction. As before, the effective viscosity is vertically averaged to find $\eta_{i+\frac{1}{2}, j+\frac{1}{2}}$ (cf. Eq. B2).

$$
\begin{aligned}
& \eta_{i-\frac{1}{2}, j+\frac{1}{2}}\left(\frac{\partial u}{\partial x}\right)_{i-\frac{1}{2}, j+\frac{1}{2}}=\frac{1}{2 \Delta x} \eta_{i-\frac{1}{2}, j+\frac{1}{2}} .
\end{aligned}
$$

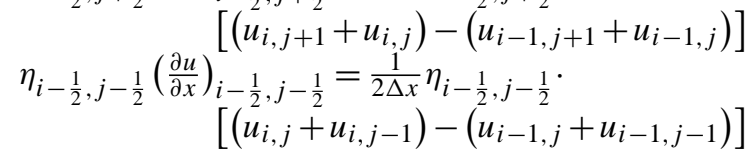

These two terms are averaged in $x$-direction after the $y$ derivatives are determined via centred differences.

$$
\begin{aligned}
\frac{\partial}{\partial y}\left[\eta\left(\frac{\partial u}{\partial x}\right)\right]_{i, j}=\frac{1}{2} \frac{\partial}{\partial y}\{[ & {\left.\left[\left(\frac{\partial u}{\partial x}\right)\right]_{i-\frac{1}{2}, j+\frac{1}{2}}+\left[\eta\left(\frac{\partial u}{\partial x}\right)\right]_{i+\frac{1}{2}, j+\frac{1}{2}}\right\}=} \\
=\frac{1}{4 \Delta y \Delta x}[ & {\left[u _ { i , j } \left(\eta_{i-\frac{1}{2}, j+\frac{1}{2}}+\eta_{i+\frac{1}{2}, j-1 \frac{1}{2}}-\right.\right.} \\
& \left.\eta_{i-\frac{1}{2}, j-\frac{1}{2}} \eta_{i+\frac{1}{2}, j+\frac{1}{2}}\right)+ \\
& u_{i, j+1}\left(\eta_{i-\frac{1}{2}, j+\frac{1}{2}}-\eta_{i+\frac{1}{2}, j+\frac{1}{2}}\right)+ \\
& u_{i, j-1}\left(\eta_{i+\frac{1}{2}, j-\frac{1}{2}}-\eta_{i-\frac{1}{2}, j-\frac{1}{2}}\right)+ \\
& u_{i+1, j}\left(\eta_{i+\frac{1}{2}, j+\frac{1}{2}}-\eta_{i+\frac{1}{2}, j-\frac{1}{2}}\right)+ \\
& u_{i-1, j}\left(\eta_{i-\frac{1}{2}, j-\frac{1}{2}}-\eta_{i-\frac{1}{2}, j+\frac{1}{2}}\right)+ \\
& u_{i-1, j-1} \eta_{i-\frac{1}{2}, j-\frac{1}{2}}+u_{i+1, j+1} \eta_{i+\frac{1}{2}, j+\frac{1}{2}}- \\
& \left.u_{i+1, j-1} \eta_{i+\frac{1}{2}, j-\frac{1}{2}}-u_{i-1, j+1} \eta_{i-\frac{1}{2}, j+\frac{1}{2}}\right]
\end{aligned}
$$

Given these two examples, forms $\Omega(y, \eta, u)$ and $\Psi(x, y, \eta, u)$ follow by simple substitution.

\section{B2 Vertically non-equidistant mesh}

With a generally non-equally spaced vertical grid, a finite difference scheme for the two operators becomes more elaborate. First we introduce some notation for the chosen vertical spacing $\xi_{k}$.

$$
\Delta \zeta_{k+\frac{1}{2}}=\zeta_{k+1}-\zeta_{k} \text { or } \Delta \zeta_{k}=\frac{1}{2}\left(\zeta_{k+1}-\zeta_{k-1}\right)
$$

In the vertical the Newton formula yields the following interpolation for a second order approximation of function values $f_{k}$.

$$
f_{k}=\frac{\Delta \zeta_{k+\frac{1}{2}}}{2 \cdot \Delta \zeta_{k}} \cdot f_{k-\frac{1}{2}}+\frac{\Delta \zeta_{k-\frac{1}{2}}}{2 \cdot \Delta \zeta_{k}} \cdot f_{k+\frac{1}{2}}
$$

This weighting can be used to retrieve a finite difference approximation for the vertical derivative based on a centred scheme.

$$
\begin{aligned}
\left(\frac{\partial f}{\partial \zeta}\right)_{k}= & \frac{\Delta \zeta_{k-\frac{1}{2}}}{\Delta \zeta_{k+\frac{1}{2}} \cdot \Delta \zeta_{k}} \cdot f_{k+\frac{1}{2}}-\frac{\Delta \zeta_{k+\frac{1}{2}}}{\Delta \zeta_{k-\frac{1}{2}}} \cdot \Delta \zeta_{k} \cdot f_{k-\frac{1}{2}}+ \\
& \frac{\Delta \zeta_{k+\frac{1}{2}}-\Delta \zeta_{k-\frac{1}{2}}}{\Delta \zeta_{k+\frac{1}{2}} \cdot \Delta \zeta_{k-\frac{1}{2}}} \cdot f_{k}
\end{aligned}
$$

Assuming equal vertical spacing $\left(\Delta \zeta_{k}=\Delta \zeta_{k+\frac{1}{2}}=\right.$ $\left.\Delta \zeta_{k-\frac{1}{2}}=\Delta \zeta\right)$ this form reduces to the normal centred difference approximation by removing the last term.

\section{B2.1 In-line derivative $\Omega(\xi, \eta, u)$}

In analogy to the equally spaced grid but applying Eqs. (B6)(B8), this in-line derivative is computed as follows. For the in-line staggered point, the inner derivatives can directly be calculated using centred differences. However, the term $\eta \partial_{\xi} u$ will also be needed on the regular grid and for this we use Eq. (B8).

$$
\begin{gathered}
\eta_{i, j, k+\frac{1}{2}} \cdot\left(\frac{\partial u}{\partial \zeta}\right)_{i, j, k+\frac{1}{2}}=\frac{1}{\Delta \zeta}{ }_{k+\frac{1}{2}} \eta_{i, j, k+\frac{1}{2}} \cdot\left\{u_{i, j, k+1}-u_{i, j, k}\right\} \\
\eta_{i, j, k} \cdot\left(\frac{\partial u}{\partial \zeta}\right)_{i, j, k}=\frac{1}{2} \eta_{i, j, k} \cdot\left\{\frac{\Delta \zeta{ }_{k-\frac{1}{2}}}{\Delta \zeta{ }_{k+\frac{1}{2}} \Delta \zeta k} u_{i, j, k+1}-\right. \\
\left.\frac{\Delta \zeta{ }_{k+\frac{1}{2}}}{\Delta \zeta{ }_{k-\frac{1}{2}}^{\Delta \zeta k}} u_{i, j, k-1}+\frac{\Delta \zeta{ }_{k+\frac{1}{2}}-\Delta \zeta_{k-\frac{1}{2}}^{\Delta \zeta}}{\Delta \zeta} u_{i, j, k}\right\} \\
\eta_{i, j, k-\frac{1}{2}} \cdot\left(\frac{\partial u}{\partial \zeta}\right)_{i, j, k-\frac{1}{2}}=\frac{1}{\Delta \zeta}{ }_{k-\frac{1}{2}} \eta_{i, j, k-\frac{1}{2}} \cdot\left\{u_{i, j, k}-u_{i, j, k-1}\right\}
\end{gathered}
$$

Since effective viscosities are needed on respective intermediate grid points, one averages the calculated staggered values $\eta_{i+\frac{1}{2}, j+\frac{1}{2}, k+\frac{1}{2}}$ appropriately. The extra division by two appearing in the expression for $\eta_{i, j, k} \cdot\left(\partial_{\zeta} u\right)_{i, j, k}$, originates from determining the three terms in Eq. (B8) on the regular grid. Applying the same equation another time, now unmodified, one obtains a numerical approximation for $\Omega(\zeta, \eta, u)$.

$$
\begin{aligned}
& \frac{\partial}{\partial \zeta}\left(\eta\left(\frac{\partial u}{\partial \zeta}\right)\right)_{i, j, k}=\frac{\Delta \zeta_{k-\frac{1}{2}}}{2 \Delta \zeta_{k+\frac{1}{2}}^{2} \Delta \zeta k} \eta_{i, j, k+\frac{1}{2}} \cdot\left(\frac{\partial u}{\partial \zeta}\right)_{i, j, k+\frac{1}{2}}- \\
& \frac{\Delta \zeta_{k+\frac{1}{2}}}{2 \Delta \zeta^{2}-\frac{1}{2} \Delta \zeta k} \eta_{i, j, k-\frac{1}{2}} \cdot\left(\frac{\partial u}{\partial \zeta}\right)_{i, j, k-\frac{1}{2}}+ \\
& \frac{\left(\Delta \zeta_{k+\frac{1}{2}}-\Delta \zeta_{k-\frac{1}{2}}\right)}{2 \Delta \zeta_{k+\frac{1}{2}}{ }^{2}{ }_{k-\frac{1}{2}}} \eta_{i, j, k} \cdot\left(\frac{\partial u}{\partial \zeta}\right)_{i, j, k} \\
& =u_{i, j, k+1} \cdot\left\{\frac{\Delta \zeta{ }_{k-\frac{1}{2}}}{\underbrace{2 \Delta \zeta^{2}}_{k+\frac{1}{2}} \eta_{i, j, k+\frac{1}{2}}}+\right. \\
& \left.\frac{\left(\Delta \zeta_{k+\frac{1}{2}}-\Delta \zeta k-\frac{1}{2}\right)}{\left.2 \Delta \zeta^{2}\right)_{k+\frac{1}{2}} \Delta \zeta k} \eta_{i, j, k}\right\}+ \\
& u_{i, j, k-1} \cdot\left\{\frac{\Delta \zeta+\frac{1}{2}}{\underbrace{2 \Delta \zeta^{2}}_{k-\frac{1}{2}} \Delta \zeta_{k}} \eta_{i, j, k-\frac{1}{2}}+\right. \\
& \left.\frac{\left(\Delta \zeta_{k+\frac{1}{2}}-\Delta \zeta_{k-\frac{1}{2}}\right)}{{\underset{k-\frac{1}{2}}{2 \Delta \zeta^{2}} \Delta \zeta k}_{k}} \eta_{i, j, k}\right\}+
\end{aligned}
$$




$$
\begin{aligned}
& u_{i, j, k} \cdot\left\{\begin{array}{c}
\frac{\left(\Delta \zeta_{k+\frac{1}{2}}-\Delta \zeta_{k-\frac{1}{2}}\right.}{2 \Delta \zeta^{2}{ }_{k+\frac{1}{2}} \Delta \zeta^{2}{ }_{k-\frac{1}{2}}} \eta_{i, j, k}- \\
\frac{\Delta \zeta_{k+\frac{1}{2}}}{2 \Delta \zeta^{2}{ }_{k-\frac{1}{2}} \Delta \zeta k} \eta_{i, j, k-\frac{1}{2}}-\frac{\Delta \zeta_{k-\frac{1}{2}}}{2 \Delta \zeta^{2}{ }_{k+\frac{1}{2}} \Delta \zeta k} \eta_{i, j, k+\frac{1}{2}}
\end{array}\right\}
\end{aligned}
$$

Again, assuming uniform spacing in the vertical, this equation reduces to the form $\Omega(x, \eta, u)$ in Eq. (B3).

\section{B2.2 Cross derivative $\Psi(x, \xi, \eta, u)$}

This function's inner derivative is computed with a centred difference scheme. But in analogy to the equidistant form, the velocities are beforehand averaged in $x$-direction. This provides values for $\partial_{x}\left(\eta \cdot \partial_{\zeta} u\right)_{i+\frac{1}{2}, j, k+\frac{1}{2}}$ at each centre of grid box $x, \zeta$-faces.

$$
\begin{aligned}
\eta_{i-\frac{1}{2}, j, k+\frac{1}{2}}\left(\frac{\partial u}{\partial \zeta}\right)_{i-\frac{1}{2}, j, k+\frac{1}{2}}=\frac{1}{2 \Delta \zeta_{k+\frac{1}{2}}} \eta_{i-\frac{1}{2}, j, k}+ \\
\frac{1}{2} \cdot\left\{\left(u_{i, j, k+1}-u_{i, j, k}\right)+\left(u_{i-1, j, k+1}-u_{i-1, j, k}\right)\right\}
\end{aligned}
$$

To compute the final derivative on the regular grid (cf. Fig. 1), vertical averaging becomes necessary followed by approximating the horizontal derivative by a centred difference.

$$
\begin{aligned}
& \frac{\partial}{\partial x}\left(\eta\left(\frac{\partial u}{\partial \zeta}\right)\right)_{i, j, l}= \frac{1}{4 \Delta \Delta \Delta \zeta k}\left[u_{i+1, j, k-1} \cdot\left\{-\frac{\Delta \zeta_{k+\frac{1}{2}}}{\Delta \zeta_{k-\frac{1}{2}}} \eta_{i+\frac{1}{2}, j, k-\frac{1}{2}}\right\}+\right. \\
& u_{i-1, j, k-1} \cdot\left\{\frac{\Delta \zeta_{k+\frac{1}{2}}}{\Delta \zeta_{k-\frac{1}{2}}} \eta_{i-\frac{1}{2}, j, k-\frac{1}{2}}\right\}+ \\
& u_{i+1, j, k+1} \cdot\left\{\frac{\Delta \zeta_{k-\frac{1}{2}}}{\Delta \zeta_{k+\frac{1}{2}}} \eta_{i+\frac{1}{2}, j, k+\frac{1}{2}}\right\}+ \\
& u_{i-1, j, k+1} \cdot\left\{-\frac{\Delta \zeta_{k+\frac{1}{2}}}{\Delta \zeta_{k-\frac{1}{2}}} \eta_{i-\frac{1}{2}, j, k+\frac{1}{2}}\right\}+ \\
& u_{i+1, j, k} \cdot\left\{\frac{\Delta \zeta_{k+\frac{1}{2}}}{\Delta \zeta_{k-\frac{1}{2}}} \eta_{i+\frac{1}{2}, j, k-\frac{1}{2}}-\frac{\Delta \zeta_{k-\frac{1}{2}}}{\Delta \zeta_{k+\frac{1}{2}}} \eta_{i+\frac{1}{2}, j, k+\frac{1}{2}}\right\}+ \\
& u_{i-1, j, k} \cdot\left\{-\frac{\Delta \zeta_{k+\frac{1}{2}}}{\Delta \zeta_{k-\frac{1}{2}}} \eta_{i-\frac{1}{2}, j, k-\frac{1}{2}}+\frac{\Delta \zeta_{k-\frac{1}{2}}}{\Delta \zeta_{k+\frac{1}{2}}} \eta_{i-\frac{1}{2}, j, k+\frac{1}{2}}\right\}+ \\
& u_{i, j, k+1} \cdot\left\{\frac{\Delta \zeta_{k-\frac{1}{2}}}{\Delta \zeta_{k+\frac{1}{2}}} \eta_{i+\frac{1}{2}, j, k+\frac{1}{2}}-\frac{\Delta \zeta_{k+\frac{1}{2}}}{\Delta \zeta_{k-\frac{1}{2}}} \eta_{i-\frac{1}{2}, j, k+\frac{1}{2}}\right\}+ \\
& u_{i, j, k-1} \cdot\left\{-\frac{\Delta \zeta_{k+\frac{1}{2}}}{\Delta \zeta_{k-\frac{1}{2}}} \eta_{i+\frac{1}{2}, j, k-\frac{1}{2}}+\frac{\Delta \zeta_{k+\frac{1}{2}}}{\Delta \zeta_{k-\frac{1}{2}}} \eta_{i-\frac{1}{2}, j, k-\frac{1}{2}}\right\}+ \\
& u_{i, j, k} \cdot\left\{\frac{\Delta \zeta_{k+\frac{1}{2}}}{\Delta \zeta_{k-\frac{1}{2}}} \eta_{i+\frac{1}{2}, j, k-\frac{1}{2}}-\frac{\Delta \zeta_{k+\frac{1}{2}}}{\Delta \zeta_{k-\frac{1}{2}}} \eta_{i-\frac{1}{2}, j, k-\frac{1}{2}}+\right. \\
&\left.\left.\frac{\Delta \zeta_{k-\frac{1}{2}}}{\Delta \zeta_{k+\frac{1}{2}}} \eta_{i-\frac{1}{2}, j, k+\frac{1}{2}}-\frac{\Delta \zeta_{k-\frac{1}{2}}}{\Delta \zeta_{k+\frac{1}{2}}} \eta_{i+\frac{1}{2}, j, k+\frac{1}{2}}\right\}\right]
\end{aligned}
$$

\section{B2.3 Cross derivative $\Psi(\zeta, x, \eta, u)$}

Swapping the sequence of derivatives affects the numerical realisation of $\Psi(\zeta, x, \eta, u)$ for the non-equidistant vertical spacing. This means one cannot just swap the indices in Eq. (B12) to retrieve the respective coefficients. Anyway, the derivation is in analogy to the previous operator (see also Fig. 1) and one finds the following approximation.

$$
\begin{aligned}
& \frac{\partial}{\partial x}\left(\eta\left(\frac{\partial u}{\partial \zeta}\right)\right)_{i, j, k}=\frac{1}{4 \Delta x \Delta \zeta_{k} \Delta \zeta_{k+\frac{1}{2}} \Delta \zeta_{k-\frac{1}{2}}}
\end{aligned}
$$

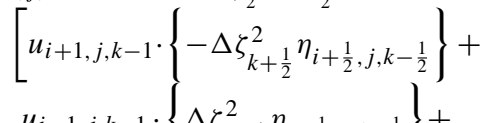

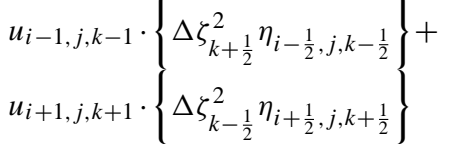

$$
\begin{aligned}
& u_{i-1, j, k+1} \cdot\left\{-\Delta \zeta_{k-\frac{1}{2}}^{2} \eta_{i-\frac{1}{2}, j, k+\frac{1}{2}}\right\}+ \\
& u_{i+1, j, k} \cdot\left\{\Delta \zeta_{k-\frac{1}{2}}^{2} \eta_{i+\frac{1}{2}, j, k+\frac{1}{2}}-\Delta \zeta_{k+\frac{1}{2}}^{2} \eta_{i+\frac{1}{2}, j, k-\frac{1}{2}}+\right. \\
& \left.2 \cdot\left(\Delta \zeta_{k+\frac{1}{2}}-\Delta \zeta_{k-\frac{1}{2}}\right) \Delta \zeta_{k} \eta_{i+\frac{1}{2}, j, k}\right\}+ \\
& u_{i-1, j, k} \cdot\left\{\Delta \zeta_{k+\frac{1}{2}}^{2} \eta_{i-\frac{1}{2}, j, k-\frac{1}{2}}-\Delta \zeta_{k-\frac{1}{2}}^{2} \eta_{i-\frac{1}{2}, j, k+\frac{1}{2}}+\right. \\
& \left.2 \cdot\left(\Delta \zeta_{k+\frac{1}{2}}-\Delta \zeta_{k-\frac{1}{2}}\right) \Delta \zeta_{k} \eta_{i-\frac{1}{2}, j, k}\right\}+ \\
& \begin{array}{l}
u_{i, j, k+1} \cdot\left\{\begin{array}{l}
\Delta \zeta_{k-\frac{1}{2}}^{2} \eta_{i-\frac{1}{2}, j, k+\frac{1}{2}}-\Delta \zeta_{k+\frac{1}{2}}^{2} \eta_{i+\frac{1}{2}, j, k+\frac{1}{2}} \\
u_{i, j, k-1} \cdot\left\{\zeta_{k+\frac{1}{2}}^{2} \eta_{i+\frac{1}{2}, j, k-\frac{1}{2}}-\Delta \zeta_{k-\frac{1}{2}}^{2} \eta_{i-\frac{1}{2}, j, k-\frac{1}{2}}\right.
\end{array}\right\}+
\end{array} \\
& u_{i, j, k} \cdot\left\{\Delta \zeta_{k+\frac{1}{2}}^{2} \eta_{i+\frac{1}{2}, j, k-\frac{1}{2}}-\Delta \zeta_{k+\frac{1}{2}}^{2} \eta_{i-\frac{1}{2}, j, k-\frac{1}{2}}+\right. \\
& \Delta \zeta_{k-\frac{1}{2}}^{2} \eta_{i-\frac{1}{2}, j, k+\frac{1}{2}}-\Delta \zeta_{k-\frac{1}{2}}^{2} \eta_{i+\frac{1}{2}, j, k+\frac{1}{2}}+ \\
& \left.\left.2 \cdot\left(\Delta \zeta_{k+\frac{1}{2}}-\Delta \zeta_{k-\frac{1}{2}}\right) \Delta \zeta_{k}\left(\eta_{i-\frac{1}{2}, j, k}-\eta_{i+\frac{1}{2}, j, k}\right)\right\}\right]
\end{aligned}
$$

Since the vertical derivative is computed with vertical weighting (see Eq. B8), terms showing differences in adjacent layers thicknesses $\Delta \zeta_{k+1 / 2}-\Delta \zeta_{k-1 / 2}$ appear in this equation. These terms are highly sensitive to the actual structure of the used vertical discretisation. Note that the two cross derivatives $\Psi(\zeta, x, \eta, u)$ and $\Psi(x, \zeta, \eta, u)$ are numerically not the same.

\section{Appendix C Analysis on large scales}

In this section an argument is derived for local extrema in the velocity field of the higher-order model on large-scale ice sheets. Extrema in the surface velocity field are ultimately linked to extrema in bedrock topography.

For the following analysis, the force balance Eq. (11) is reduced to 2 dimension. This allows rewriting the elliptic operator

$\mathrm{P}[u(x, \zeta)]=f(x)$

with

$$
\begin{aligned}
\mathrm{P}[u(x, \zeta)] & =4 \cdot \Omega(x, \eta, u)+4 a_{x} \cdot\{\Psi(x, \zeta, \eta, u)+ \\
& \Psi(\zeta, x, \eta, u)\}+\left(4 a_{x}^{2}+a_{z}^{2}\right) \cdot \Omega(\zeta, \eta, u) \\
f(x) & =\rho g \cdot \partial_{x} s
\end{aligned}
$$

As surface boundary condition serves a reduced form of Eq. (7)

$4\left(\partial_{x^{\prime}} u+a_{x} \cdot \partial_{\zeta} u\right) \cdot \partial_{x^{\prime}} s+\frac{1}{H} \cdot \partial_{\zeta} u=0$ 
Since a large-scale ice sheet is considered where the length scale of perturbations is long compared to the ice thickness (as in experiment $\mathrm{A}$ on $160 \mathrm{~km}$ ), the following assumptions are made. At first the effective viscosity is assumed to be a constant $\eta(x, \zeta)=\eta_{0}$, which simplifies the double mixed derivatives in the two operators.

$\mathrm{P}[u(x, \zeta)] \approx 4 \eta_{0} \cdot \partial_{x}^{2} u+8 a_{x} \eta_{0} \cdot \partial_{x \zeta}^{2} u+\left(4 a_{x}^{2}+a_{z}^{2}\right) \eta_{0} \cdot \partial_{\zeta}^{2} u$

This equation illustrates the elliptic character of the underlying partial differential equation. Ellipticity signifies an inequality for the three factors, which is here automatically fulfilled.

$4 \eta_{0} \cdot\left(4 a_{x}^{2}+a_{z}^{2}\right) \eta_{0}-\frac{1}{4}\left(8 a_{x} \eta_{0}\right)^{2}=4 \eta_{0}^{2} a_{z}^{2}>0$

The second assumption for large-scale ice sheets is that vertical plane shearing is well described by the shallow ice approximation. This links vertical velocity gradients to the surface slope.

$\partial_{\zeta} u \approx 2 A_{0}(\rho g)^{n} H^{n+1} \zeta^{n} \cdot\left|\partial_{x} s\right|^{n-1} \partial_{x} s$

With these assumptions we focus on the near surface region, i.e. $\zeta=\varepsilon \ll 1$. The second vertical derivative and the coefficient $a_{x}$ become.

$$
\begin{aligned}
& \left.\partial_{\zeta}^{2} u\right|_{\varepsilon} \approx 2 A_{0}(\rho g)^{n} H^{n+1} n \varepsilon^{n-1} \cdot\left|\partial_{x} s\right|^{n-1} \partial_{x} s \\
& \left.a_{x}\right|_{\varepsilon}=\frac{1}{H}\left(\partial_{x} s-\varepsilon \cdot \partial_{x} H\right)=\frac{1}{H}\left((1-\varepsilon) \cdot \partial_{x} s-\varepsilon \cdot \partial_{x} b\right)
\end{aligned}
$$

Near the surface the upper boundary condition (C3) is applicable, linking vertical and horizontal derivatives. Together with Eqs. (C7) the operator takes the following form.

$$
\begin{gathered}
\mathrm{P}[u(x, \zeta)] \approx\left\{\frac{4 \eta_{0}}{H}\left((1-\varepsilon) \cdot \partial_{x} s-\varepsilon \cdot \partial_{x} b\right)-\frac{\eta_{0}}{H \cdot \partial_{x} s}\right\} \cdot \partial_{x \zeta}^{2} u+ \\
2 n A_{0} \eta_{0}(\rho g)^{n}\left(4 a_{x}^{2}+a_{z}^{2}\right) H^{n+1} \varepsilon^{n-1} \cdot\left|\partial_{x} s\right|^{n-1} \partial_{x} s
\end{gathered}
$$

For a constitutive equation using a flow index of three, terms with exponents higher than 1 in $\varepsilon$ are neglected. This finally provides

$$
\begin{aligned}
\mathrm{P}[u(x, \zeta)] & \approx \frac{\eta_{0}}{H}\left(4(1-\varepsilon) \cdot \partial_{x} s+4 \varepsilon \cdot \partial_{x} b-\frac{1}{\partial_{x} s}\right) \cdot \partial_{x \zeta}^{2} u \\
& =\rho g \cdot \partial_{x} s
\end{aligned}
$$

With the assumption that the vertical part of the double derivative is well described by the shallow shelf approximation, this derivative does not change sign. Thus changes in signs due to the bedrock topography have to be compensated for by the horizontal velocity derivative as long as the right hand side and thus the surface slope does not change sign.

Applied to the main flow line in experiment $\mathrm{A}$ of the ISMIP-HOM model intercomparison (Pattyn et al., 2008) on a $160 \mathrm{~km}$ domain, the surface slope is a constant value, while ice thickness varies sinusoidal together with bedrock topography. Thus all terms of the sum are constant except for the bedrock topography. Where the ice depth is deepest, the bed slope changes from negative to positive sign causing the $x$ velocity gradient to change likewise in a close vicinity. This results in a maximal velocity since the vertical derivative part is supposed to show a negative sign. Consequently in this case of low aspect ratio, velocity extrema are ultimately linked to bed topography.

Acknowledgements. The research was funded mainly by the Commission of the European Communities through the Marie Curie Research Training Networks program Network of Ice sheet and Climate Evolution (NICE) under contract number MRTN-CT-2006036127, by the Research Foundation - Flanders (FWO) project NEEM-B on Nested modelling of the Greenland ice sheet in support of the dating and the interpretation of the NEEM ice core record. This work was also supported by funding from the ice 2 sea programme from the European Union 7th Framework Programme, grant number 226375. Ice2sea contribution number 053. We want to also express our gratitude to the reviewers Torsten Albrecht, Frank Pattyn but especially to Jed Brown whose review comments improved the clarity and quality of the presented work.

Let me again express my thanks for your commitment and the considerable work you have done.

Edited by: C. Ritz

\section{References}

Albrecht, T., Martin, M., Haseloff, M., Winkelmann, R., and Levermann, A.: Parameterization for subgrid-scale motion of ice-shelf calving fronts, The Cryosphere, 5, 35-44, doi:10.5194/tc-5-352011, 2011.

Benn, D. I., Warren, C. R., and Mottram, R. H.: Calving processes and the dynamics of calving glaciers, Earth-Sci. Rev., 82, 143179, doi:10.1016/j.earscirev.2007.02.002, 2007.

Berman, A. and Plemmons, R. J.: Nonnegative Matrices in the Mathematical Sciences, Society for Industrial and Applied Mathematics (SIAM), ISBN:978-0898713213, 1994.

Blatter, H.: Velocity and stress fields in grounded glaciers: a simple algorithm for including deviatoric stress gradients, J. Glaciol., 41, 333-344, 1995.

Bueler, E. and Brown, J.: Shallow shelf approximation as a "sliding law" in a thermomechanically coupled ice sheet model, J. Geophys. Res.-Earth, 114(F03008), 21 pp., doi:10.1029/2008JF001179, 2009.

Colinge, J. and Blatter, H.: Stress and velocity fields in glaciers: Part I. Finite-difference schemes for higher-order glacier models, J. Glaciol., 44), 448-456, 1998.

De Smedt, B., Pattyn F., and De Groen, P.: Using the unstable manifold correction in a Picard iteration to solve the velocity field in higher-order ice-flow models, J. Glaciol., 56, 257-261, doi:10.3189/002214310791968395, 2010.

Dukowicz, J. K., Price, S. F., and Lipscomb, W. H.: Consistent approximations and boundary conditions for ice-sheet dynamics from a principle of least action, J. Glaciol., 56, 480-496, doi:10.3189/002214310792447851, 2010.

Ferziger, J. H. and Perić, M.: Computational Methods for Fluid Dynamics, Springer Verlag, 3, ISBN:978-3540594345, 2002.

Fowler, A. C. and Larson, D. A.: The uniqueness of steady state flows of glaciers and ice sheets, Geophys. J. Roy. Astr. S., 63, 333-345, doi:10.1111/j.1365-246X.1980.tb02624.x, 1980. 
Gagliardini, O., Duran, G., Zwinger, T., Hindmarsh, R. C. A., and Le Meur, E.: Coupling of ice-shelf melting and buttressing is a key process in ice-sheet dynamics, Geophys. Res. Lett., 37, 24 pp., doi:10.1029/2010GL043334, 2010.

Goldberg, D., Holland, D. M., and Schoof, C.: Grounding line movement and ice shelf buttressing in marine ice sheets, J. Geophys. Res.-Earth, 114, 23 pp., doi:10.1029/2008JF001227, 2009.

Harlow, F. and Welch, J.: Numerical calculation of time-dependent viscous incompressible flow of fluid with free surface, Phys. Fluids, 8, 2182-2189, doi:10.1063/1.1761178, 1965.

Hindmarsch, R. C. A.: A numerical comparison of approximations to the Stokes equations used in ice sheet and glacier modelling, J. Geophys. Res.-Earth, 109, F01012, doi:10.1029/2003JF000065, 2004.

Hindmarsch, R. C. A.: The role of membrane-like stresses in determining the stability and sensitivity of the Antarctic ice sheets: back pressure and grounding line motion, Philos. T. Roy. Soc. A., 364, 1733-1767, doi:10.1098/rsta.2006.1797, 2006.

Hindmarsch, R. C. A. and Payne, A. J.: Time-step limits for stable solutions of the ice-sheet equation, Ann. Glaciol., 23, 74-85, 1996.

Hutter, K.: Theoretical glaciology. Material science of ice and the mechanics of glaciers and ice sheets, Reidel, Dordrecht, Netherlands, ISBN:978-9027714732, 1983.

Huybrechts, P. and de Wolde, J.: The dynamic response of the Greenland and Antarctic ice sheets to multiple-century climatic warming, J. Climate, 12, 2169-2188, doi:10.1175/15200442(1999)012<2169:TDROTG>2.0.CO;2, 1999.

Johnson, J. and Fastook, J. L.: Northern Hemisphere glaciation and its sensitivity to basal melt water, Quatern. Int., 95-96, 65-74, doi:10.1016/S1040-6182(02)00028-9, 2002.

Jouvet, G., Huss, M., Blatter, H., Picasso, M., and Rappaz, J.: Numerical simulation of Rhonegletscher from 1824 to 2100 , J. Comput. Phys., 228, 6426-6439, doi:10.1016/j.jcp.2009.05.033, 2009.

LeVeque, R. J.: Finite Difference Methods for Ordinary and Partial Differential Equations, SIAM, Philadelphia, ISBN: 978$0898716290,2007$.

MacAyeal, D. R.: Large-Scale Ice Flow Over a Viscous Basal Sediment: Theory and Application to Ice Stream B, Antarctica, J. Geophys. Res.-Solid, 94, 4071-4087, doi:10.1029/JB094iB04p04071, 1989.

Mattheij, R. M. M., Rienstra, S. W., and ten Thije Boonkamp, J. H. M.: Partial differential equations: modeling, analysis, computation, SIAM, Philadelphia, ISBN:978-0898715941, 2005.

Morland, L. W.: Unconfined ice-shelf flow, in: Dynamics of the West Antarctic ice sheet, dited by: van der Veen, C. J. and Oerlemans, J., Kluwer Academic Publishers, 99-116, ISBN:9789027723703, 1986.

Morland, L. W. and Johnson, I. R.: Steady Motion of Ice Sheets, J. Glaciol., 25, 229-246, 1980.

Manguoglu, M., Sameh, A. H., Saied, F., Tezduyar, T. E., and Sathe, S.: Preconditioning Techniques for Nonsymmetric Linear Systems in the Computation of Incompressible Flows, J. Appl. Mech., 76, 7 pp., doi:10.1115/1.3059576, 2009.

Otero, J., Navarro, F. J., Martin, C., Cuadrado, M. L., and Corcuera, M. I.: A three-dimensional calving model: numerical experiments on Johnsons Glacier, Livingston Island, Antarctica, J. Glaciol., 56, 200-214, doi:10.3189/002214310791968539,
2010.

Pattyn, F.: A new three-dimensional higher-order thermomechanical ice sheet model: Basic sensitivity, ice stream development, and ice flow across subglacial lakes, J. Geophys. Res.-Sol. Earth, 108, 2382, doi:10.1029/2002JB002329, 2003.

Pattyn, F., Perichon, L., Aschwanden, A., Breuer, B., de Smedt, B., Gagliardini, O., Gudmundsson, G. H., Hindmarsh, R. C. A., Hubbard, A., Johnson, J. V., Kleiner, T., Konovalov, Y., Martin, C., Payne, A. J., Pollard, D., Price, S., Rückamp, M., Saito, F., Soucček, O., Sugiyama, S., and Zwinger, T.: Benchmark experiments for higher-order and full-Stokes ice sheet models (ISMIPÜHOM), The Cryosphere, 2, 95-108, doi:10.5194/tc-2-952008, 2008.

Pimentel, S., Flowers, G. E., and Schoof, C. G.: A hydrologically coupled higher-order flow-band model of ice dynamics with a Coulomb friction sliding law, J. Geophys. Res.-Earth, 115, F04023, doi:10.1029/2009JF001621, 2010.

Pollard, D. and DeConto, R. M.: Modelling West Antarctic ice sheet growth and collapse through the past five million years, Nature, 458, 7236, 329-332, doi:10.1038/nature07809, 2009.

Press, W. H., Teukoslky, S. A., Vetterling, W. T., and Flannery, B. P.: Numerical Recipes in Fortran, Second Edition: The Art of Scientific Computing, Volume 1 of Fortran Numerical Recipes, Cambridge University Press, New York, ISBN:978-0521430647, 2003.

Pritchard, H. D., Arthern, R. J., Vaughan, D. G., and Edwards, L. A.: Extensive dynamic thinning on the margins of the Greenland and Antarctic ice sheets, Nature, 461, 7266, doi:10.1038/nature08471, 2009.

Ritz, C., Rommelaere, V., and Dumas, C.: Modeling the evolution of Antarctic ice sheet over the last 420,000 years: Implications for altitude changes in the Vostok region, J. Geophys. Res.Atmos., 106, 31943-31964, doi:10.1029/2001JD900232, 2001.

Saad, Y. and Schultz, M. H.: GMRES: A Generalized Minimal Residual Algorithm for Solving Nonsymmetric Linear Systems, SIAM, Philadelphia, 7, 856-869, doi:10.1137/0907058, 1986.

Schoof, C.: A variational approach to ice streams flow, J. Fluid. Mech., 556, 227-251, doi:10.1017/S0022112006009591, 2006.

Schoof, C.: Ice sheet grounding line dynamics: Steady states, stability, and hysteresis, J. Geophys. Res., 112, F03S28, doi:10.1029/2006JF000664, 2007.

Schoof, C. and Hindmarsh, R. C. A.: Thin-Film Flows with Wall Slip: An Asymptotic Analysis of Higher Order Glacier Flow Models, Q. J. Mech. Appl. Math., 63, 73-114, doi:10.1093/qjmam/hbp025, 2010.

Shepherd, A. and Wingham, D.: Recent sea-level contributions of the Antarctic and Greenland ice sheets, Science, 315, 15291532, doi:10.1126/science.1136776, 2007.

Sleijpen, G. L. G. and van Gijzen, M. B.: Exploiting BiCGstab $(l)$ Strategies to Induce Dimension Reduction, SIAM, Philadelphia, 32, 2687-2709, doi:10.1137/090752341, 2010.

Sonneveld, P. and Van Gijzen, M. B.: IDR(s): a family of simple and fast algorithms for solving large nonsymmetric systems of linear equations, SIAM, Philadelphia, 31, 1053-1062, doi: $10.1137 / 070685804,2008$.

Trottenberg, U., Oosterlee, C. W. and Schüller, A.: Multigrid, Academic Press, ISBN: 978-0127010700, 2001.

Truffer, M. and Fahnestock, M.: Rethinking ice sheet time scales, Science, 315, 1508-1510, doi:10.1126/science.1140469, 2007. 
Tulaczyk, S., Kamb, W. B., and Engelhardt, H. F.: Basal mechanics of Ice Stream B, West Antarctica 1. Till mechanics, J. Geophys. Res.-Sol. Earth, 105, 463-481, 2000.

van Gijzen, M. B. and Sonneveld, P.: An elegant IDR(s) variant that efficiently exploits bi-orthogonality properties, Technical Report 10-16, Department of Applied Mathematical Analysis, Delft University of Technology, Delft, 2010.

Van Der Veen, C. J.: A Numerical Scheme for Calculating Stresses and Strain Rates in Glacier, Math. Geol., 21, 363-377, doi:10.1007/BF00893696, 1989.

Van Der Veen, C. J.: Fundamentals of Glacier Dynamics, edited by: Balkema, A. A., Rotterdam, Netherlands, ISBN: 9789054104704, 1999.

Van der Vorst, H. A.: Bi-CGSTAB: A Fast and Smoothly Converging Variant of Bi-CG for the Solution of Nonsymmetric Linear Systems, SIAM, Philadelphia, doi:10.1137/0913035, 1992.

Vaughan, D. G. and Arthern, R.: Why is it hard to predict the future of ice sheets?, Science, 315, 1503-1504, doi:10.1126/science.1141111, 2007.
Veldman, A. E. P. and Rinzema, K.: Playing with nonuniform grids, J. Eng. Math., 26, 119-130, doi:10.1007/BF00043231, 1992.

Weis, M., Greve, R., and Hutter, K.: Theory of shallow ice shelves, Continuum Mech. Therm., 11, 15-50, doi:10.1007/s001610050102, 1999.

Wesseling, P. and Sonneveld, P.: Numerical Experiments with a Multiple Grid and a Preconditioned Lanczos Type Method, Lecture Notes in Mathematics 771, Springer Verlag, 543-562, 1980.

Yeung, M.-C. and Chan, T. F.: ML $(K)$ BiCGSTAB: A BiCGSTAB Variant based on Multiple Lanczos Starting Vectors, SIAM, Philadelphia, 21, 1263-1290, doi:10.1137/S1064827597321581, 1999.

Zwinger, T., Greve, R., Gagliardini, O., Takayuki, S., and Lyly, M.: A full Stokes-flow thermo-mechanical model for firn and ice applied to the Gorshkov crater glacier, Kamchatka, Ann. Glaciol., 45, 29-37, doi:10.3189/172756407782282543, 2007. 